\title{
1 A causal role for estradiol in human reinforcement learning
}

2

3

4 Sebastijan Veselic ${ }^{1,2,3 *}$, Gerhard Jocham ${ }^{4}$, Christian Gausterer $^{5}$, Bernhard Wagner ${ }^{6}$, Miriam

5 Ernhoefer-Reßler ${ }^{6}$, Rupert Lanzenberger ${ }^{7}$, Claus Lamm $^{1,8}$, Christoph Eisenegger $^{1}$ \& Annabel

6

7

\section{$8 \quad$ Affiliations}

${ }^{1}$ Neuropsychopharmacology and Biopsychology Unit, Department of Cognition, Emotion, and Methods in Psychology, Faculty of Psychology, University of Vienna, Austria

${ }^{2}$ Department of Clinical and Movement Neurosciences, University College London, London, UK

${ }^{3}$ Wellcome Centre for Human Neuroimaging, University College London, London, UK

${ }^{4}$ Biological Psychology of Decision Making, Institute of Experimental Psychology, Heinrich Heine University Düsseldorf, Germany

${ }^{5}$ FDZ-Forensisches DNA Zentrallabor GmbH, Medical University of Vienna, Austria

${ }^{6}$ Laboratory for Chromatographic \& Spectrometric Analysis, FH JOANNEUM, Graz, Austria

${ }^{7}$ Department of Psychiatry and Psychotherapy, Medical University of Vienna, Vienna,

Austria,

${ }^{8}$ Vienna Cognitive Science Hub, University of Vienna, Austria

${ }^{*}$ ) corresponding authors: Sebastijan Veselic (sebastijan.veselic.18@ucl.ac.uk) and Annabel Losecaat Vermeer (annabel.losecaat.vermeer@univie.ac.at) 


\section{Abstract}

26 The sex hormone estrogen is hypothesized to play a key role in human cognition via its 27 interactions with the dopaminergic system. Work in rodents has shown that estrogen's most potent form, estradiol, impacts striatal dopamine functioning predominately via increased D1receptor signalling, while human work has suggested that high estradiol levels are associated with altered reward sensitivity. Here, we addressed two fundamental questions: 1) whether estradiol causally alters reward sensitivity in men, and 2) whether this effect of estradiol is moderated by individual variation in polymorphisms of dopaminergic genes. To test this, we performed a double-blind placebo-controlled administration study in which hundred men received either a single dose of estradiol $(2 \mathrm{mg})$ or placebo. We found that estradiol administration increased reward sensitivity, which was moderated by baseline dopamine. This was observed in choice behaviour and increased learning rates. These results confirm a causal role of estradiol in reinforcement learning in men that is moderated by the striatal dopaminergic pathway.

Keywords: Estradiol, reward processing, reinforcement learning, DAT1, COMT, Estrogen receptor 


\section{Introduction}

43 Learning which action to select based on whether the outcome of that action is rewarded or not is a fundamental capacity required for adaptive behaviour. One neuromodulator that has long been linked to this capacity, known as reinforcement learning $(R L)$, is dopamine ${ }^{1}$. More recently, an additional biological substrate that has been suggested to influence $R L$ via dopaminergic mechanisms is estrogen ${ }^{2}$.

Estrogens are a class of steroid hormones important for healthy development, with estradiol being the most prevalent and potent form ${ }^{3,4}$. Estradiol has gained traction as a compound that may impact human reward processing by amplifying dopamine signalling via the D1 receptor ${ }^{2}$. The evidence for this hypothesis comes from two lines of work. From human work, we know that fluctuations in circulating estradiol levels are correlated with differences in midbrain BOLD responses, a key area where dopamine is released ${ }^{5-7}$. Furthermore, a recent administration study in women showed that increased salivary estradiol levels led to larger reward prediction errors in the nucleus accumbens ${ }^{8}$. From rodent work, we know that manipulating estradiol levels affects the striatal dopamine system in various ways that are best characterised as a net increase in overall dopamine signalling predominantly via the D1 receptor ${ }^{9-14}$.

If increased estradiol levels in humans influence reward processing by increasing reward sensitivity through amplified dopamine signalling via the D1 receptor, then it should be possible to relate its effects to that of dopamine in $\mathrm{RL}^{1,15-17}$. Within this field, dopamine agonists and antagonists are often administered to better understand the mechanistic role of dopamine in $R L^{18-24}$. Complementing it with pharmacogenetics, where the effect of genetic variation on the administered drug is considered, has refined our understanding of their relationship ${ }^{19,22,25-}$ ${ }^{27}$. That is, causally manipulating dopamine levels in humans affects performance in $R L$ tasks ${ }^{27}$ with these effects depending on individual differences in baseline dopamine levels ${ }^{18}$.

Two key polymorphisms that lead to variation in baseline dopamine levels by impacting dopamine synthesis capacity and transmission are the dopamine transporter (DAT1) and 
catechol-O-methyltransferase (COMT) gene ${ }^{19,24,25}$. Variation in the VNTR polymorphism of DAT1 and the vallis8met polymorphism of COMT correlates with performance on working memory and $R \mathbf{L}$ in humans ${ }^{19,24,25,28,29}$. Therefore, if estradiol influences reward processing through dopaminergic mechanisms, then variation in the DAT1 and COMT genotype should moderate the effect of estradiol. Furthermore, we would predict it should be moderated by traits related to human reward sensitivity such as the ones measured by the BIS/BAS questionnaire ${ }^{30-33}$.

Despite abundant evidence from rodent research showing a clear relationship between estradiol and dopamine, evidence in humans have been less conclusive. Namely, it has been shown that high endogenous estradiol levels were associated with increased ${ }^{5,7}$ as well as decreased ${ }^{34}$ performance on a variety of cognitive tasks that may have recruited different neural mechanisms. Thus, it remains unclear whether administering estradiol influences reward processing as would be expected by amplified D1-receptor signalling. Furthermore, although previous work on humans provided important insights, it had certain shortcomings such as small sample sizes, correlational study designs, and a lack of accounting for baseline differences in dopamine; for exceptions see ${ }^{8,34,35} 25,36$. All studies thus far have also focussed on female samples who have, on average, higher endogenous levels of estradiol compared to men ${ }^{37}$. These aspects are important for being able to establish a more precise role of estradiol in human reward processing which, therefore, remains an open question (for review $\left.\operatorname{see}^{2}\right)$.

The aim of the present pharmacogenetic study was to address these gaps and investigate whether estradiol administration increases reward sensitivity in men and whether this effect is moderated by baseline differences in dopamine. To test this, we used a probabilistic RL task (Fig. 1A) where subjects had to choose between two options on each trial in order to maximize their earnings. Moreover, we aimed at providing a more conclusive and precise account of a dopamine-dependent basis of action through excluding several other candidate explanations (i.e. gene polymorphisms) that could have given rise to the obtained results and have so far been unaddressed in the literature (see Supplementary Materials). 
We hypothesized that estradiol administration would influence reward processing by increasing reward sensitivity observed through subjects' choice behaviour ${ }^{8}$. We further predicted this would be revealed through computational modelling. Finally, we predicted that the behavioural and computational effects would be moderated by the DAT1 polymorphism, as observed in previous dopamine administration work ${ }^{19,26}$.

\section{Methods and materials}

\section{Subjects}

We tested one hundred healthy young men between 19 and 34 years $\left(M_{\text {age }}=24.86, S D=\right.$ 3.53) with a body mass index (BMI) between 19.3 and $31.5(M=24.45, S D=2.86)$. Our screening procedure was based on previous work where pharmacokinetic data for a single 2 mg estradiol dose in topical form was obtained ${ }^{38}$. Our sample size was in line with previous recommendations for the field ${ }^{2}$. The study procedure was performed in accordance with the Declaration of Helsinki and approved by the Medical Ethics Committee of the University of Vienna (1918/2015).

\section{Measurement Instruments}

\section{Questionnaires}

We assessed self-reported mood (German Multidimensial Mood State Questionnaire; $\mathrm{MDBF}^{39}$ ), individuals' impulsiveness (Barratt Impulsiveness Scale; BIS-1140), and behavioural activation and inhibition (BIS/BAS ${ }^{41}$ ). Both BIS/BAS and BIS-11 scores have been previously

117 found to correlate with reward learning ${ }^{42-45}$ with the BIS/BAS specifically being related to reward sensitivity ${ }^{30,31}$. In addition, we probed subjects' beliefs about estradiol (e.g. whether they believed they received estradiol or a placebo, how certain they were about their belief, and whether they had noticed any subjective changes). 
122

123

\section{Hormone concentrations}

We collected hormone samples via passive drool and stored them at -30 degrees Celsius. Saliva samples were analyzed for estrone and estradiol using gas chromatography tandem mass spectrometry (GC-MS/MS) and hydrocortisone including testosterone with liquid chromatography tandem mass spectrometry (LCMS/MS).

\section{Genotyping}

We collected DNA using sterile cotton buccal swabs (Sarstedt AG, Germany) and extracted it by applying the QIAamp DNA Mini kit (Qiagen, Germany). Repeat length polymorphisms (AR(CAG), AR(GGN), DAT1(VNTR), ERa(TA) and $E R B(C A))$ were investigated by PCR with fluorescent-dye-labeled primers and capillary electrophoresis. The single base primer extension (SBE) method also known as minisequencing was applied for the typing of single nucleotide polymorphism (SNP) variants (Val158Met) in the COMT gene.

\section{Experimental Tasks}

Reinforcement Learning: We used a well-established probabilistic reinforcement learning task with two options ${ }^{17}$. The task consisted of 500 trials, with a 10 second pause after the first 250 trials. There was no choice time-out. The two options had independently varying reward probabilities generated by Gaussian random walks (Fig. 1). Importantly, both options could be correct (i.e. rewarding - yellow frame) or incorrect (i.e. non-rewarding - reward frame) on any trial. Subjects received feedback for the chosen (thick frame) and unchosen (thin frame) option. Each correct choice was rewarded with 5 eurocents and added to their cumulative balance. Subjects also saw a bar fill up as they chose the correct option. Once the bar filled up, a $1 €$ coin was presented next to the bar indicating they had added $1 €$ to their cumulative balance.

Working memory capacity: We used an adapted version of the standard N-BACK task ${ }^{25}$ with four conditions in total (0-BACK, 1-BACK, 2- BACK, 3-BACK). Each condition block had 20 trials which included $20 \%$ target, $65 \%$ nontarget, and $15 \%$ lure trials. Subjects were 
presented with a sequence of letters one-by-one. For each letter, they had to decide if the current letter was the same as the one presented $N$ trials ago by pressing "R", in case it was not the same they had to press "O".

Procedure

Subjects screened for BMI, a history of psychiatric disorders, concurrent involvement in other psychopharmacological studies, and chronic physical injuries, arrived to the lab on two separate days. On the first session, at $4.00 \mathrm{pm}$, we collected subjects' responses on a battery of questionnaires, their DNA with a buccal swab for genotyping, and measured their BMI and body fat using a body composition monitor (Omron BF51). MDBF-A, BIS-11, and BIS/BAS. Twenty minutes after arrival, we obtained the first saliva sample ( $\mathrm{T} 1)$ to assess baseline hormone concentrations, followed by the N-BACK task. They were randomly assigned estradiol or placebo in a double-blind manner and self-administered a topical transparent gel containing either $2 \mathrm{mg}$ of estradiol or a placebo. We waited two hours to allow estradiol levels to peak based on a previously established procedure ${ }^{38}$. Fifteen minutes prior to the behavioural testing, subjects filled out MDBF-B, BIS-11, and provided a second saliva sample (T2).

The first behavioural task was the probabilistic reinforcement learning task, followed by three other tasks that were not the focus of this publication. After the behavioural testing, we probed subjects' beliefs about the treatment and the tasks. At the end of the study, each subject was paid in accordance to their performance. 


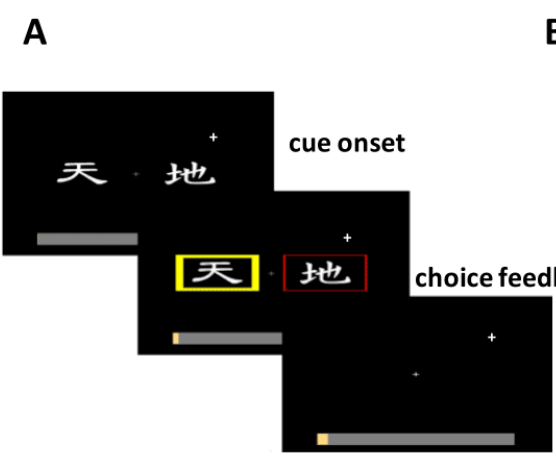

B $\stackrel{+}{\stackrel{E}{*}}$

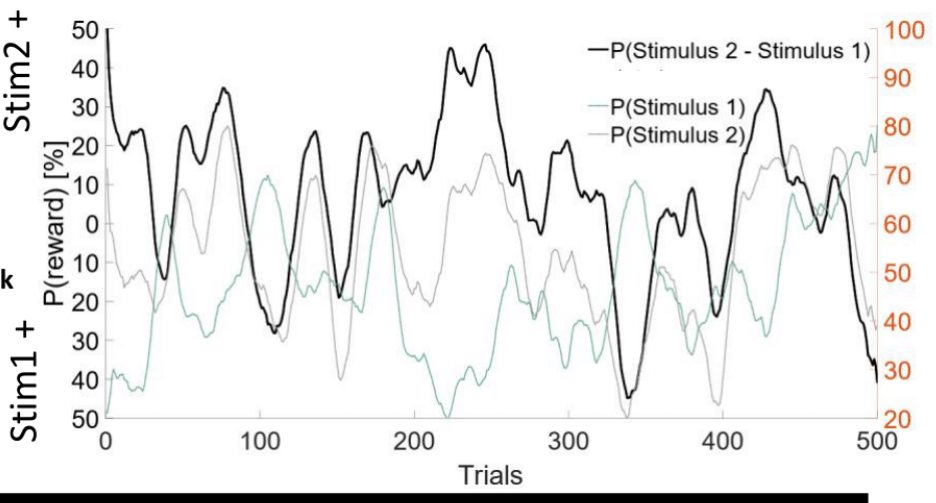

C

Fig. 1 A) Outline of a trial of the RL task. Each trial started by the presentation of two options (henceforth option A and option B). Subjects were required to choose one of these options. After they made a choice, subjects were presented with feedback, with the chosen option indicated by a thicker frame and the not chosen option by a thinner frame. A yellow frame indicated the rewarded option, whereas a red frame indicated the unrewarded option. Importantly, both options A and B could yield a reward or no reward on the same trial. B) The probability of reward upon choice for each option (green and gray lines), which were determined by two independent random Gaussian walks, with the probability shown in percent on the right $y$-axis in orange. The black line shows the relative probability of reward for one option over the other, which corresponds to the difference in reward probability for option A and option $B$. On trials where the black line is reaching the top half of the $y$-axis, option A was more rewarding, and vice versa. C) The timeline of the test session. Values in brackets denote minutes from the onset of the test session. We first collected consent and questionnaire data, which was followed by a baseline saliva sample (T1) and the N-BACK task. After administration of estradiol or placebo, subjects were required to rest for two hours before we collected the second saliva sample (T2) and assessed subjects' mood and impulsivity via questionnaires. The RL task began 120 minutes post-administration. This was followed by three other cognitive tasks that are not the focus of the current paper. At the end of the test session, we probed subjects' beliefs about the drug, the experiment, and debriefed them. 
195

\section{Statistical analysis}

\section{Behavioural analysis}

To determine the effect of estradiol on choice behaviour, we examined the cumulative difference in the options subjects chose and the percentage of trials on which there was a significant difference in the chosen option. As a measure of family-wise error control, we used permutation testing and Fisher-z-transformations.

We also computed choice accuracy because we predicted that if estradiol would influence reward processing by increasing reward sensitivity, this would result in increased accuracy and be moderated by differences in striatal dopamine functioning (i.e. through the DAT1 genotype) ${ }^{26}$. Due to the same reasoning we computed how many trials subjects would pick the same option if they were rewarded for that option on trial $t$ (staying). Accuracy and staying were statistically evaluated with general linear models.

In addition to the above metrics, we computed subjects' choice autocorrelation. With this, we tested our hypothesis that increased reward prediction errors would, by definition, upregulate saliency of recent events ${ }^{8}$. Behaviourally, this would mean subjects' recent choices should influence what they will choose next more. We computed the relative contribution of choices made from $n-1$ to $n-7$ trials back (lags) on the current choice. We then compared the relative contribution of all previous choices (pure choice autocorrelation) and specifically choices when they were rewarded (choice autocorrelation as a function of reward). We then performed independent samples $t$-tests on individual lags that were $z$-scored within subject to assess statistical significance.

\section{Computational modelling}

To account for behaviour within a computational framework and relate our findings to the field exploring dopamine and its role in $\mathrm{RL}$, we used computational modelling. We fit a series of $\mathrm{Q}$ learning models with softmax choice rules. The winning model included a learning rate for positive and negative prediction errors, a temperature parameter, and an irreducible noise 
parameter (see Supplementary materials for model selection and parameter recovery). Our main hypothesis was that if estradiol would increase reward sensitivity, that should be captured by the learning rate, reflecting how strongly new information will be weighed and incorporated into the subjects' subjective values.

\section{Results}

Our sample was matched for age, height, visceral, and abdominal fat, BMI, working memory, self-reported impulsivity, behavioural inhibition and approach, and mood. As a manipulation check of our administration protocol, estradiol concentrations were significantly elevated in subjects who had received estradiol compared to placebo after $(W=1545,95 \% \mathrm{Cl}[0.03$, 1.87], $p<.05$ ), but not before administration (baseline: $W=1498,95 \% \mathrm{Cl}[-0.05,1.03], p=$ .09) and subjects' beliefs about whether they had received estradiol or placebo did not correlate with the actual received drug $(r=0.02, p=.82$; for further details see Supplementary Materials).

\section{Estradiol administration increases reward sensitivity}

Our first hypothesis was that estradiol administration would increase reward sensitivity. Because reward size was kept constant, the only difference across trials was whether a choice was rewarded or not. Therefore, we predicted that increased reward sensitivity would be reflected in a systematic difference in the options subjects would choose across trials compared to placebo. To test this, we computed the average probability per group to select option $A / B$ (i.e. expected chosen option) on each trial (Fig. 2A), subtracted these traces from each other, and plotted the cumulative choice difference across trials (Fig. 2B).

The cumulative choice difference in the expected chosen option between both groups surpassed what would be expected by chance. This was tested by comparing the above results to the $99.9^{\text {th }}$ percentile of a null distribution (see Methods and materials) indicating what would be expected by chance $\left(M_{\text {last trial }}=53.48 \%\right.$, $Z_{\text {last trial }}=8.44, p<.001$, threshold for 
$24799.9^{\text {th }}$ percentile of the null: $46.20 \%$, Fig. 2B). The observed cumulative choice difference

248 remained significant when collapsed across time $(M=25.72 \pm 0.69 \%, z=5.80, p<.001$,

249 threshold for $99.9^{\text {th }}$ percentile of the null: $M=21.02 \%$, Fig. $\left.2 \mathrm{C}\right)$. When we traced trials that

250 contributed most to the cumulative difference, we observed a statistically significant difference

251 in what both groups chose on $7.6 \%$ of trials (black dots in Fig. 2A). In other words, estradiol

252 administration caused subjects to choose a different option on $7.6 \%$ of trials as compared to

253 placebo $\left(z=5.37, p<.001\right.$, threshold for $99.9^{\text {th }}$ percentile of null distribution: $\left.6.4 \%\right)$.

However, estradiol administration did not only increase reward sensitivity in choice

behaviour as described above. When subjects rated how strongly they observed the changing of reward probabilities of both options throughout the task on a scale from 1 to 100 during debriefing, those receiving estradiol explicitly reported that they observed reward probabilities across trials to change more strongly $\left(t_{(78.495)}=2.15,95 \% \mathrm{Cl}=[0.855,22.61], p=0.035, d=\right.$ $\left.0.48, B F_{10}=3.28\right)$. 

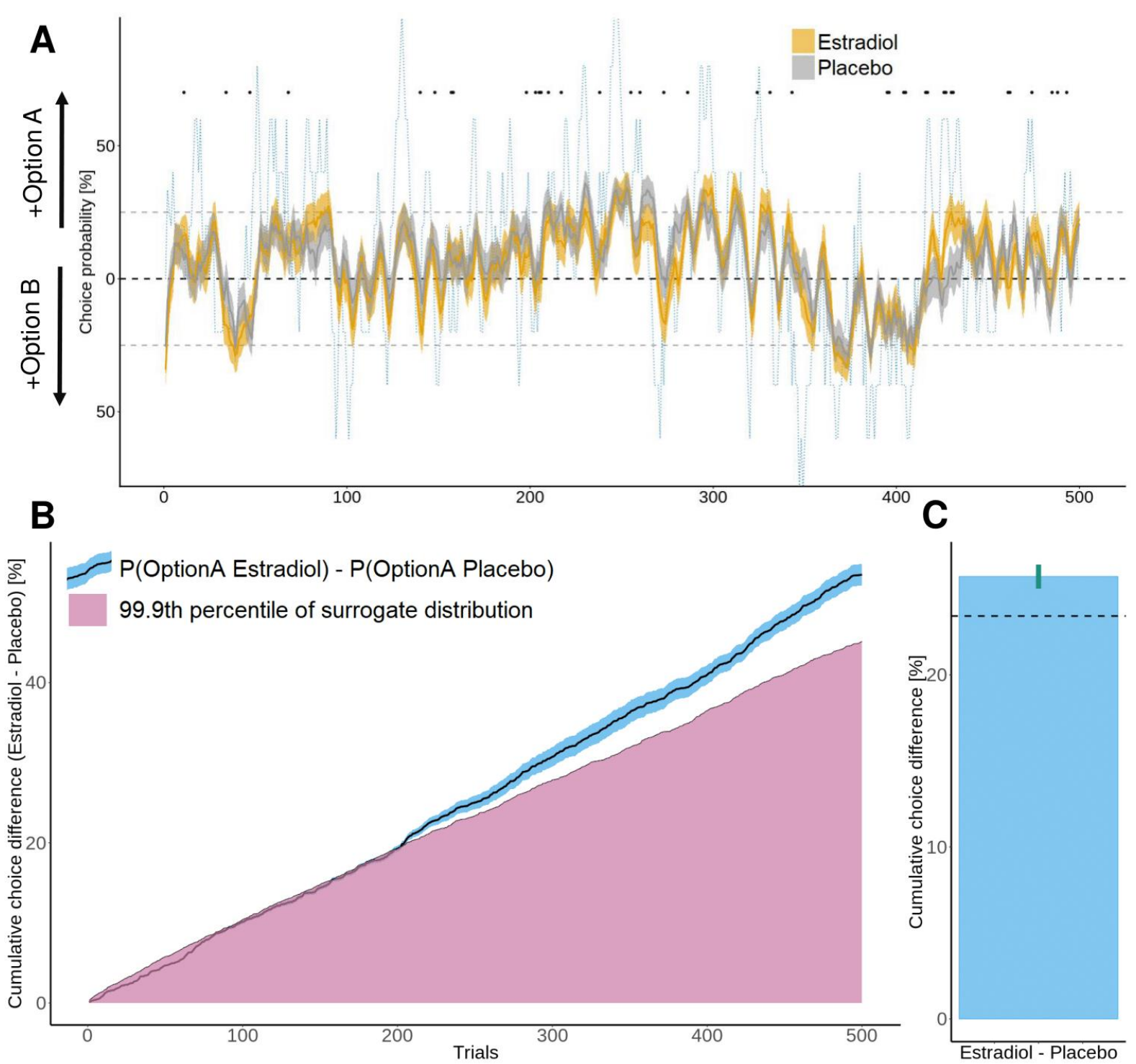

Fig. 2 A) Relative choice probability for choosing option A (top of y-axis) vs. choosing option B (bottom of $y$-axis) for the estradiol (orange) and placebo (gray) group. Solid thick lines represent trial mean, shaded areas around the thick lines denote standard errors of the mean. The blue dotted line denotes the relative reward probability which was computed from the probability of option $A$ (top of $y$-axis) minus probability of option $B$ (bottom of $y$-axis). Horizontal gray dotted lines represent where subjects were on average $25 \%$ more likely to select option A (upper line) or option B (lower line). All time-series traces were smoothed with a 5 -trial moving average for visual purposes. The black dots indicate trials where there was a statistically significant difference $(p<.05)$ between the estradiol and placebo group. The number of significant trials was compared to a null distribution (see Methods and materials). B) Cumulative choice difference between the estradiol and placebo group over trials compared to the 99.9th ${ }^{\text {th }}$ percentile null distribution. The thick black line is the difference between the orange and gray lines presented in figure $\mathbf{A}$, and the blue shaded area is the corresponding difference between the standard errors in $\mathbf{A}$. The dark orange area denotes the space in which differences are not significant. Conversely, separation between the lines indicate statistical significance. C) Mean cumulative choice difference between the estradiol and placebo group collapsed across trials. The dashed line represents the mean cumulative choice difference of the $100^{\text {th }}$ percentile of the null distribution. Error bars indicate standard error of the mean. 

reward responsiveness

281

282

283

284

285

286

287

Following the observed systematic choice difference between both groups, we investigated whether this was reflected in group differences in accuracy. Both groups were equally accurate $\left(M_{\text {Estradiol }}=57.30 \pm 6.91, M_{\text {Placebo }}=56.80 \pm 7.09, t_{(97.94)}=0.36, p=.72\right.$, Fig. $\left.3 \mathrm{~A}\right)$, and responded equally fast $\left(M_{\text {Estradiol }}=0.61 \mathrm{sec} \pm 0.11, M_{\text {Placebo }}=0.62 \mathrm{sec} \pm 0.09, t_{(95.55)}=0.46, p=.65\right)$.

However, we predicted that the effect of estradiol on accuracy will be moderated by individual differences in baseline striatal dopamine, as other work has shown interactive effects between task performance and dopamine-related genes ${ }^{18,46}$. In our study, we used the DAT1 polymorphism as an index of striatal dopamine with the $9 / 10$ and $10 / 10$ genotypes being associated with high and low striatal dopamine, respectively ${ }^{47}$.

A general linear model revealed an interaction between drug administration and DAT1 genotype on accuracy $\left(F_{(1,69)}=4.10, p=.047, \Omega^{2}=0.037\right.$, Fig. 3B) while controlling for covariates (see Supplementary Materials). Pairwise comparisons revealed that estradiol administration increased accuracy in subjects with the 9/10 genotype (i.e. high striatal dopamine levels; $M=60.00 \pm 5.36$ ) compared to those with a $10 / 10$ genotype (i.e. low striatal dopamine levels; $10 / 10$ DAT1, $M=56.00 \pm 6.51 ; t_{(39.60)}=2.14,95 \% \mathrm{Cl}[0.21,7.63], p=.04, d$ $\left.=0.66, B F_{10}=3.16\right)$, while this difference did not exist in the placebo group $(9 / 10$ genotype: $M$ $=57.21 \pm 6.60 ; 10 / 10$ genotype: $\left.M=56.90 \pm 6.34 ; t_{(30.01)}=0.15, p=.88\right)$. However, estradiol administration did not improve accuracy in subjects with the $9 / 10$ genotype relative to placebo $\left(9 / 10: t_{(28.48)}=1.35, p=.19 ; 10 / 10: t_{(40.11)}=1.79,95 \% C l[-0.41,6.77], p=.08, d=0.55, B F_{10}\right.$ $=1.92)$. related to reward responsiveness such as the one measured by BIS/BAS ${ }^{30,31,33}$. Indeed, when we accounted for subjects' reward responsiveness (Fig 3C), we found that those who received estradiol (vs. placebo) were more accurate $\left(\beta=24.17 \pm 11.14, F_{(1,85)}=4.7, p=0.03, \Omega^{2}=\right.$ 
$0.008)$, which was further moderated by reward responsiveness $\left(F_{(1,85)}=4.6, p=0.03, \Omega^{2}=\right.$ 0.036) while controlling for covariates. That is, estradiol enhanced accuracy specifically in subjects who were less reward responsive $(r=-0.52, p<.001)$ with no such correlation in the placebo group $(r=-0.16, p=0.27)$. This result further supports the hypothesis that estradiol administration increased reward sensitivity by enhancing striatal reward prediction errors that increased the saliency of each trial ${ }^{8}$. In our task, this hypothesis would predict more reward responsive subjects to switch between the choice options more frequently. Indeed, we found that within the estradiol group, there was a significant positive correlation between reward responsiveness and switching $(r=0.37, p=0.009)$ that was only trending in subjects who received placebo $(r=0.26, p=0.07)$. Importantly, the degree of switching negatively predicted subjects' task accuracy $\left(F_{(1,98)}=69.38, p<.001, \Omega^{2}=0.41\right)$ across both groups, explaining why more reward responsive subjects who received estradiol were less accurate.
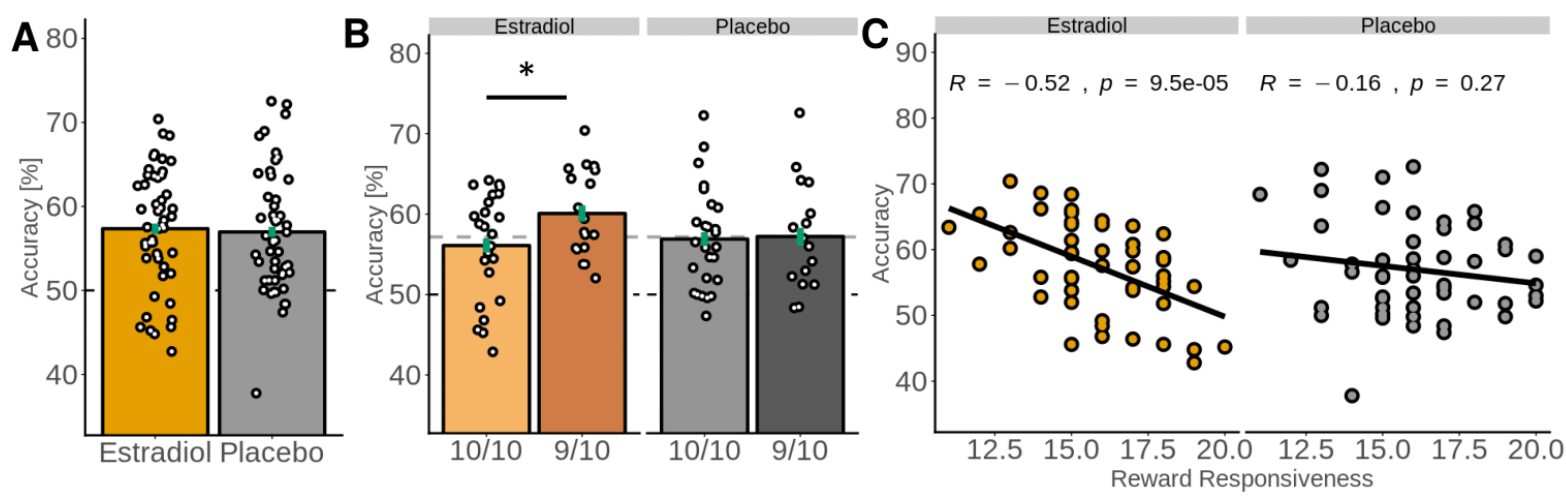

Fig. 3 A) Mean accuracy split according to drug administration. B) Mean accuracy split according to drug administration and DAT1 polymorphism. C) Accuracy was moderated by reward responsiveness. Green error bars are standard errors of the mean. Dots represent individual subjects. The horizontal dotted line represents grand mean performance collapsed across groups to show the relative change for individual subgroups. ${ }^{*} p<.05$

\section{Increased reward sensitivity is observed in increased learning rates}

Given our observation that estradiol increased reward sensitivity (Fig. 2), the interactive effect with DAT1 and reward responsiveness (Fig. 3), and our hypothesis that the differences in choice behaviour might occur due to upregulated striatal prediction errors, we predicted that 
estradiol would enhance the learning of reward probabilities. In a $\mathrm{RL}$ framework this would be reflected in increased learning rates.

To compare learning rates we used the maximum a posteriori estimates of the winning model fitted in a hierarchical Bayesian way ${ }^{48}$, which included separate learning rates for positive and negative prediction errors, a temperature parameter, and an irreducible noise parameter (see Supplementary Materials for details on model selection and parameter recovery).

This model revealed that estradiol administration increased the learning rates for positive and negative prediction errors compared to placebo ( $\alpha_{\text {Positive: }} M_{\text {Estradiol }}=0.47 \pm 0.22$, $M_{\text {Placebo }}=0.37 \pm 0.24, t_{(97.51)}=2.36,95 \% C l[0.017,0.19], p=.02, d=0.47, B F_{10}=4.77 ; \alpha_{\text {Negative }}$ : $M_{\text {Estradiol }}=0.45 \pm 0.31, M_{\text {Placebo }}==0.27 \pm 0.25, t_{(93.55)}=3.2,95 \% \mathrm{Cl}[0.068,0.29], p=.002, d=$ 0.64, $B F_{10}=35.03$, Fig. $4 \mathrm{~A}$ ). We also compared both parameters by computing $95 \%$ Highest Density Interval estimates ${ }^{49}$ (Fig. 4D) with stronger evidence in favour of the negative learning rate $95 \% \mathrm{HDI}[0.04,0.39]$ being higher in subjects who received estradiol compared to the positive learning rate $95 \% \mathrm{HDI}[-0.04,0.32]$. Contrary to our expectations, the observed main effect of estradiol was not moderated by the DAT1 polymorphism in case of either learning rate ( $\alpha_{\text {Positive }}: F_{(1,80)}=0.24, p=.89$, $\alpha_{\text {Negative }}: F_{(1,80)}=0.12, p=.73$ ).

Finally, to provide construct validation for the obtained parameters, we correlated them with the BIS/BAS Drive subscale measuring motivation for goal-directed behaviour ${ }^{40}$. We found that both positive and negative learning rates were weakly correlated in subjects who received estradiol ( $\alpha_{\text {Positive: }} r=0.3, p=0.03$, $\alpha_{\text {Negative: }} r=27, p=0.06$ ) but not who received placebo ( $\alpha_{\text {Positive: }}: r=-0.10, p=0.47, \alpha_{\text {Negative }}: r=-.09, p=0.53$ ). 

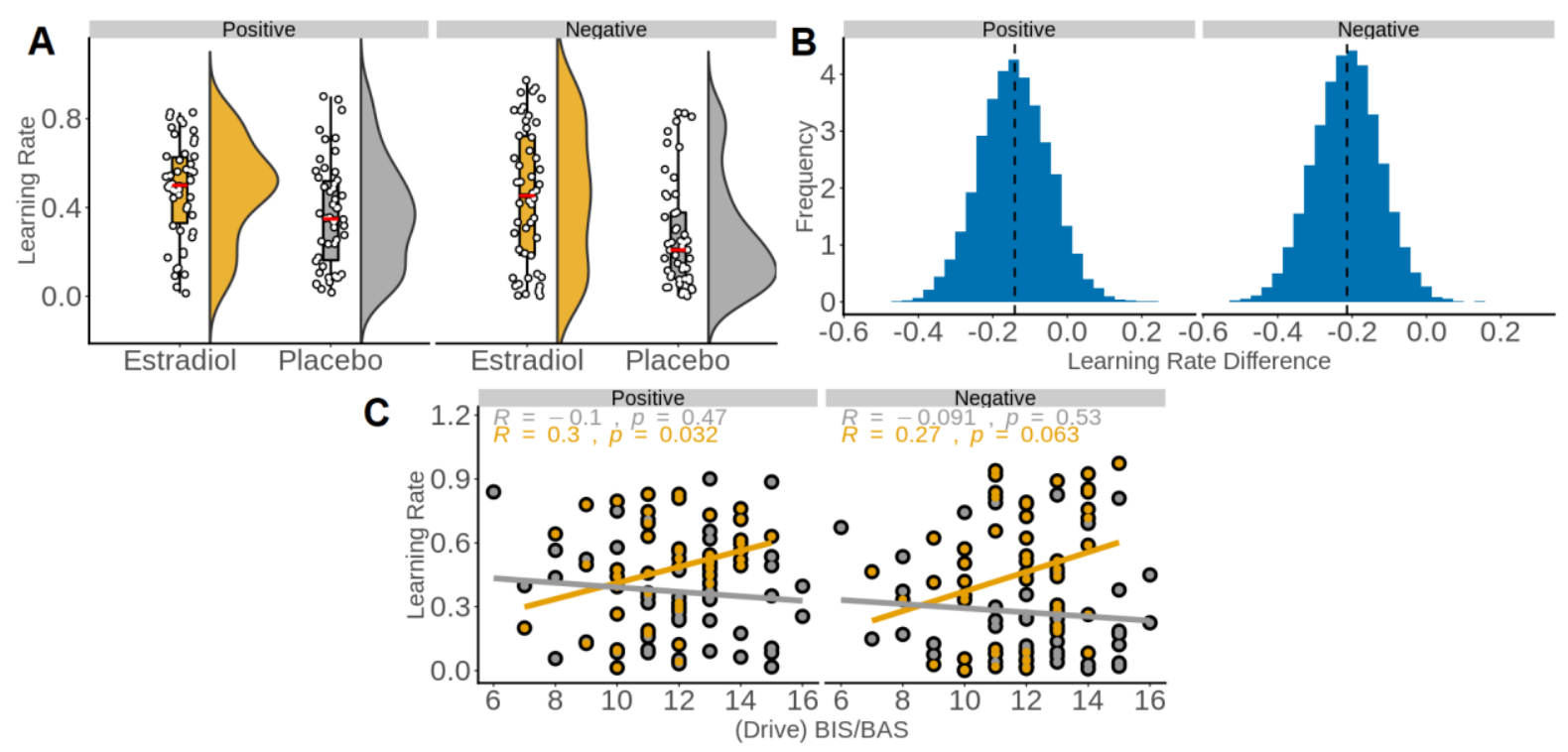

Fig. 4 A) Learning rates by drug treatment. Individual dots represent subjects. The red bar represents the median, the box plot represents $75 \%$ of data, with the whiskers representing $1.95^{\star} \mathrm{IQR}$. B) $95 \%$ highest density interval (HDI) estimates for learning rate differences between both groups. C) Correlation between both learning rates and the drive subscale of BIS/BAS measuring motivation for goal-directed behaviour.

\section{Increased reward sensitivity is driven by differences in stay decisions, choice}

Finally, to more precisely understand the observed difference in choice behaviour between both groups and DAT1 genotype, we tested whether these differences could be attributed to differences in staying behaviour and choice autocorrelation ${ }^{24,50}$.

As would be predicted by increased accuracy in the 9/10 DAT genotype subjects, on average these subjects also chose the same option more often after being rewarded for this option before $(M=1.79 \pm 0.18$; Fig. $5 \mathrm{~A})$. This was true when comparing them to subjects with placebo who had the $9 / 10$ genotype $\left(M=1.63 \pm 0.22 ; t_{(29.05)}=2.33,95 \% \mathrm{Cl}[0.02,0.3], p=\right.$ $.03, d=0.80, B F_{10}=5.01$ ), and when comparing them to subjects who had the $10 / 10$ genotype (placebo: $t_{(41.61)}=2.22,95 \% \mathrm{Cl}[0.01,0.27], p=.03, d=0.66, B F_{10}=3.34$; estradiol: $\left(t_{(38.86)}=\right.$ $\left.2.49,95 \% C l[0.03,0.27], p=.02, d=0.64, B F_{10}=5.95\right)$. In contrast, this difference was not observed between both drug groups when not accounting for differences in DAT1 genotype $\left(t_{(97.94)}=1.10, p=0.28\right)$. In other words, the increase in accuracy by exogenously elevated 
estradiol in individuals with a 9/10 genotype was reflected in increased decisions to stay with options for which they were previously rewarded.

We then extended this metric by examining how subjects' previous choice impacted their current choice. We observed that estradiol administration caused subjects to weigh their choice on the previous trial $(n-1)$ more heavily compared to placebo (Fig. 5B), both when that choice was rewarded $\left(t_{(97)}=2.61,95 \% \mathrm{Cl}[0.073,0.538], p=0.01, d=0.52, B F_{10}=8.07\right)$ and irrespective of the outcome $\left(t_{(97)}=2.21,95 \% \mathrm{Cl}[0.034,0.639], p=.03, d=0.44, B F_{10}=3.49\right)$. However, we also observed a decrease in the weight of previous rewarded choices occurring more than one trial ago $(\mathrm{n}-4)\left(t_{(97)}=2.59,95 \% \mathrm{Cl}[0.064,0.482], p=0.01, d=0.52, B F_{10}=\right.$ 7.93). In other words, while recent rewarded choices carried more weight, choices that were rewarded further in the past carried less weight, which is in line with having higher learning rates compared to the placebo group.

When we further split this analysis according to the DAT1 genotype (Fig. 5C), we observed similar results to the ones reported above. Estradiol administration enhanced the weight of the last choice $(n-1)$ on the current choice in the $9 / 10$ subgroup $\left(t_{(32)}=2.12,95 \%\right.$ $\left.C l[0.022,1.027], p=.04, d=0.73, B F_{10}=3.44\right)$ while this was not the case in the $10 / 10$ $\operatorname{subgroup}\left(t_{(48)}=1.51, p=.14\right)$. 
A

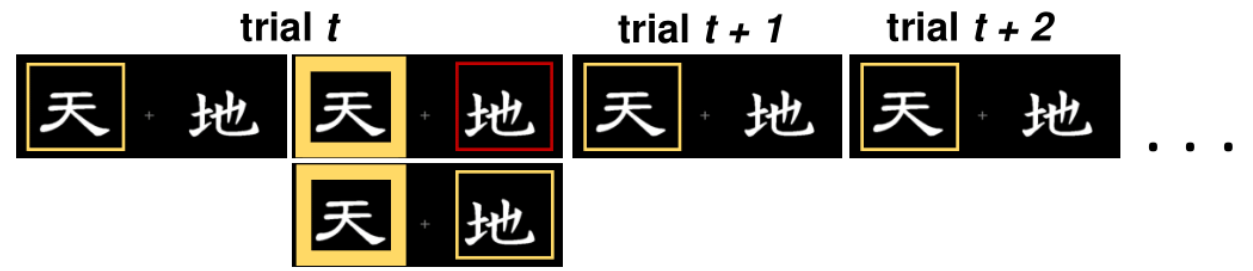

B
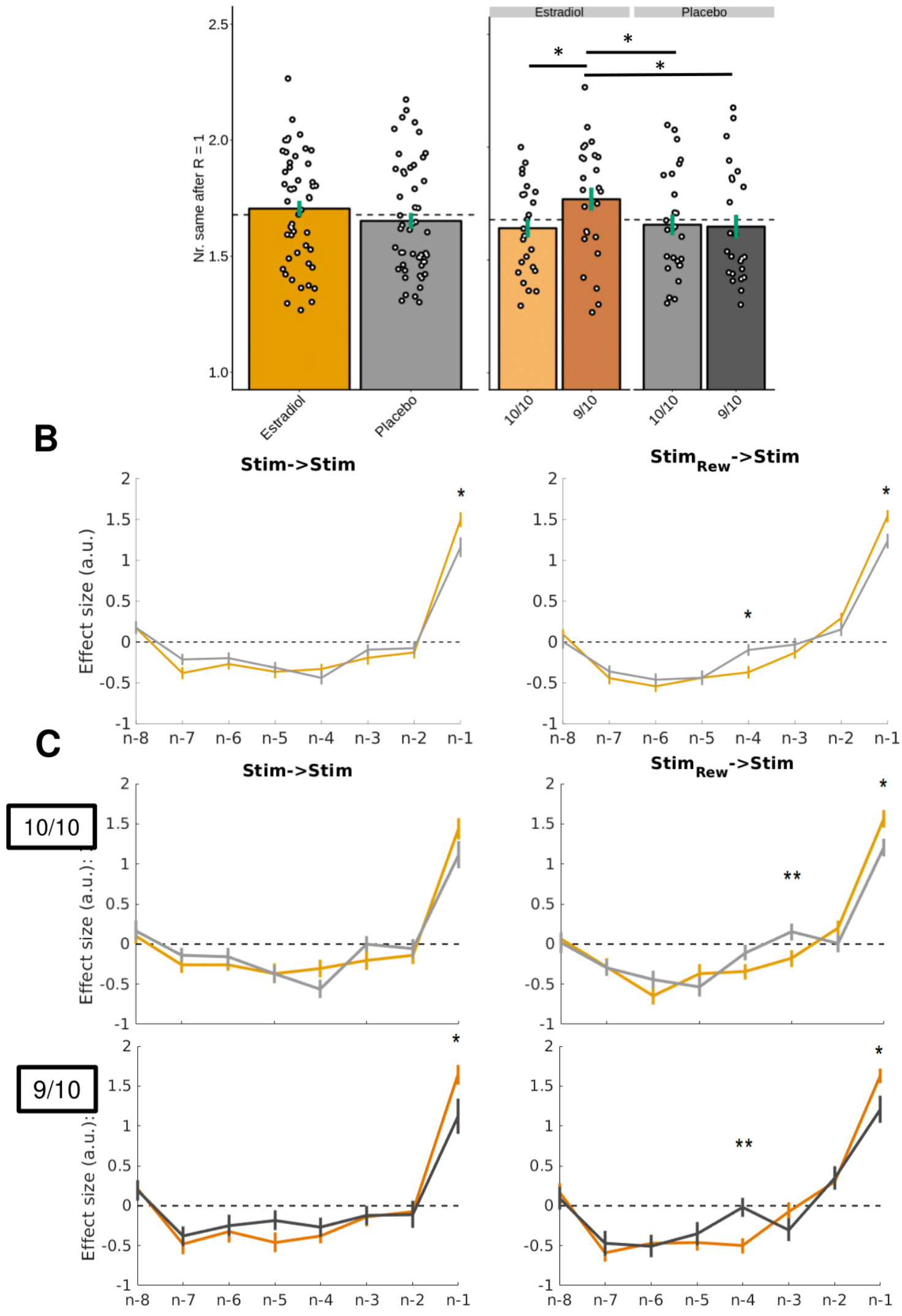
Fig. 5 A) Staying behaviour: the average number of trials the same option was selected when that choice was previously rewarded. In both plots, each dot represents a subject, the green error bar represents standard error of the mean. BC) Autocorrelation analysis showing the impact of previous choices from $1(n-1)$ to $8(n-8)$ trials ago on the current choice. The left panel shows the averaged effect for both options irrespective of whether they were previously rewarded for that choice. The right panel shows the averaged effect for both options when they were previously rewarded for that choice. Both line plots are the mean and SEM of z-scored regressor weights. B) shows the split according to estradiol and placebo while $\mathbf{C}$ ) shows a further split according to the $9 / 10$ and 10/10 DAT1 polymorphism. ${ }^{* *} p<$ $.01,{ }^{*} p<.05$.

\section{Discussion}

We examined the causal role of estradiol in reward processing in men. We show that estradiol affects reward processing and learning in men by increasing reward sensitivity, affecting choice autocorrelation, and increasing explicit awareness of changing reward probabilities. Furthermore, we show that estradiol's effect on accuracy is moderated by subjects' striatal dopaminergic functioning (DAT1 genotype) and reward responsiveness. Finally, we show that from a computational perspective, its effect can be characterised as an increase in learning rates.

First, we found that estradiol increased reward sensitivity during reinforcement learning. We observed that both groups systematically choose different options across trials, and on a subset of trials the options each group chose was statistically significantly different. Furthermore, estradiol increased choice autocorrelation for recent choices compared to placebo, while decreasing it for rewarded choice made several trials ago. These findings jointly show that by increasing reward sensitivity through upregulated striatal reward prediction errors $^{8}$, estradiol administration caused recent choices and outcomes to be more salient compared to ones occurring further in the past. This interpretation is further supported by the estradiol group having higher learning rates for both positive and negative prediction errors, and subjects explicitly reporting to have observed a higher degree of reward probability changing. Furthermore, it is supported by the effect of estradiol on accuracy being moderated by reward responsiveness. The predicted increase in reward prediction errors caused more reward responsive subjects to switch more, in turn reducing their accuracy, while this 
relationship did not exist with placebo. The key contribution of the results above is showing, for the first time, the behavioural and computational effect of estradiol administration on reinforcement learning in men.

Next, we found that the effect of estradiol on accuracy was moderated by the DAT1 genotype, replicating previous correlational studies in women ${ }^{6,75}$. This result provides evidence for the hypothesis that estradiol may act by amplifying dopamine D1 receptor signalling in humans ${ }^{2}$, as such an effect would also have been predicted by dopamine precursor administration ${ }^{27} 26,36$. To determine the robustness of this effect, we computed several behavioural metrics and Bayes factors to show converging evidence for a moderating role of DAT1. We found that estradiol administration significantly increased accuracy in subjects with the $9 / 10$ (i.e. high striatal dopamine) compared to ones with the 10/10 genotype (i.e. low striatal dopamine) and that this increase in accuracy was likely driven by increased staying behaviour. That is, subjects with high striatal dopamine chose the same option on more trials, on average, if they were previously rewarded for that choice, compared to the other subgroups. Furthermore, even when they were not rewarded, they showed stronger choice autocorrelation compared to subjects with high striatal dopamine who received a placebo.

The key contribution of the DAT1-related findings is in reconciling discrepancies of previous correlational work that have been attributed to differences in baseline dopamine levels ${ }^{7,51-53}$. That is, we show for the first time that baseline differences in dopamine, indeed, play an important role in how estradiol influences reward processing and that these need to be considered in future work aiming at better understanding their relationship. Crucially, the effects we report for accuracy and staying behaviour were not explained by other mechanistic explanations such as those related to androgen receptor functioning, androgen to estrogen conversion, or estrogen receptor functioning (see Supplementary Materials).

Our results highlight a further feature of how estradiol influences reinforcement learning. We found that estradiol improved accuracy specifically in the high striatal dopamine 
447 group which is in contrast to previous research with the dopamine precursor L-dopa where

448 decreased accuracy in such subjects was reported but increased accuracy in subjects with

449 low striatal dopamine was found ${ }^{26}$. However, it is known that dopamine precursors impact

450 behaviour in a dose-dependent manner ${ }^{54,55}$. This would imply that our estradiol dose acted

451 akin to a "low dosage" of a dopamine precursor which is supported by two estradiol

452 administration studies in women. One showing $12 \mathrm{mg}$ of estradiol (i.e. 6 times our dose) decreasing working memory performance ${ }^{34}$, which was interpreted as an overstimulation of dopaminergic transmission. With the other showing a dopamine-like dose-dependent effect of estradiol on hippocampal activity with doses between 2 and $12 \mathrm{mg} .{ }^{35}$.

In addition to the effects reported for DAT1, we also provide preliminary evidence that the effect of estradiol is moderated by COMT (see Supplementary Materials). This is in support of the hypothesis that COMT activity is inhibited through estradiol metabolites which in turn increases dopamine availability $252,5613,57$.

The current study encountered limitations. We recommend future work employing pharmacogenetics to increase their sample size. While our sample size was approximately twice as large compared to most previous work $7,25,34,36,52,53,58,59$ and in line with suggestions for the field ${ }^{2}$, we suspect that an increase in sample size, as in work on other hormones ${ }^{60,61}$, would have enabled us to make more precise claims at the level of polymorphism subgroup. As no previous estradiol administration study investigated either DAT1 or COMT, we had no basis for the minimal viable sample size, except the general recommendation in ${ }^{2}$. Our results therefore await replication in future administration work. 


\section{Conclusion}

473 In conclusion, we have shown that estradiol influences choice behaviour by increasing reward 474 sensitivity in healthy young men with distinct behavioural and computational signatures and 475 that these effects are moderated by striatal dopamine-related genes (DAT1) and personality 476 traits related to reward sensitivity. The approach and findings of this study show that 477 understanding the role of estradiol in reward processing has important implications for a better 478 understanding of the biology and neuroscience of human cognition that is moderated by genes 479 in both health and disorder.

\section{Acknowledgements}

The authors would like to thank Christina Faschinger and Isa Krol for their assistance in data collection, Nace Mikus for his help in data collection and analysis suggestions, and Lei Zhang for feedback on the manuscript. The study was supported by the Vienna Science and Technology Fund (WWTF VRG13-007).

\section{Conflict of interests}

The authors declare no conflict of interest with respect to the research, authorship, and/or publication of this article. $R L$ received travel grants and/or conference speaker honoraria within the last three years from Shire, Heel, Bruker, and support from Siemens Healthcare regarding clinical research using PET/MR. He is a shareholder of BM Health GmbH since 2019. 


\section{References}

496 1. Schultz, W., Dayan, P. \& Montague, P. R. A neural substrate of prediction and reward. Science (80-. ). 275, 1593-1599 (1997).

2. Diekhof, E. K. Estradiol and the reward system in humans. Curr. Opin. Behav. Sci. 23, 58-64 (2018).

500

3. Saldanha, C. J., Remage-Healey, L. \& Schlinger, B. A. Synaptocrine signaling: Steroid synthesis and action at the synapse. Endocr. Rev. 32, 532-549 (2011).

4. Luine, V. N. Estradiol and cognitive function: Past, present and future. Horm. Behav. 66, 602-618 (2014).

5. Thomas, J., Météreau, E., Déchaud, H., Pugeat, M. \& Dreher, J. Hormonal treatment increases the response of the reward system at the menopause transition : A counterbalanced randomized placebo-controlled fMRI study.

6. Dreher, J. et al. Menstrual cycle phase modulates reward-related neural function in women. PNAS 104, 2465-2470 (2007).

7. Diekhof, E. K. \& Ratnayake, M. Menstrual cycle phase modulates reward sensitivity and performance monitoring in young women: Preliminary fMRI evidence. Neuropsychologia 84, 70-80 (2016).

8. Bayer, J., Rusch, T., Zhang, L., Gläscher, J. \& Sommer, T. Dose-dependent effects of estrogen on prediction error related neural activity in the nucleus accumbens of healthy young women. Psychopharmacology (Berl). (2019) doi:10.1007/s00213-01905409-7.

9. Lévesque, D. \& Di Paolo, T. Rapid conversion of high into low striatal D2-dopamine receptor agonist binding states after an acute physiological dose of $17 \beta$-estradiol. 
Neurosci. Lett. 88, 113-118 (1988).

520

10. Becker, J. B. Gender Differences in Dopaminergic Function in Striatum and Nucleus Accumbens. Pharmacol. Biochem. Behav. 64, 803-812 (1999).

11. Becker, J. B. Direct effect of $17 \beta$-estradiol on striatum: Sex differences in dopamine release. Synapse 5, 157-164 (1990).

12. Pasqualini, C., Olivier, V., Guibert, B., Frain, O. \& Leviel, V. Acute Stimulatory Effect of Estradiol on Striatal Dopamine Synthesis. J. Neurochem. 65, 1651-1657 (1995).

13. Ball, P., Knuppen, R., Haupt, M. \& Breuer, H. Interactions Between Estrogens and Cateehol Amines III. Studies on the Methylation of Catechol Estrogens, Catechol Amines and other Catechols by the Catechol-O-Methyltransferase of Human Liver. $J$. Clin. Endocrinol. Metab. 34, 736-746 (1972).

14. Yoest, K. E., Quigley, J. A. \& Becker, J. B. Rapid effects of ovarian hormones in dorsal striatum and nucleus accumbens. Horm. Behav. 104, 119-129 (2018).

15. Schultz, W., Stauffer, R. W. \& Lak, A. The phasic dopamine signal maturing: from reward via behavioural activation to formal economic utility. Curr. Opin. Neurobiol. 43, $139-148(2017)$.

16. Glimcher, P. W. Understanding dopamine and reinforcement learning: the dopamine reward prediction error hypothesis. PNAS 108, 15647-54 (2011).

17. Frank, M. J., Seeberger, L. C. \& O’Reilly, R. C. By carrot or by stick: Cognitive reinforcement learning in Parkinsonism. Science (80-. ). 306, 1940-1943 (2004).

18. Cools, R. et al. Striatal Dopamine Predicts Outcome-Specific Reversal Learning and Its Sensitivity to Dopaminergic Drug Administration. J. Neurosci. 29, 1538-1543 (2009).

19. den Ouden, H. E. M. et al. Dissociable Effects of Dopamine and Serotonin on 
Reversal Learning. Neuron 80, 1090-1100 (2013).

544

20. Jocham, G., Klein, T. A. \& Ullsperger, M. Dopamine-Mediated Reinforcement Learning Signals in the Striatum and Ventromedial Prefrontal Cortex Underlie ValueBased Choices. J. Neurosci. 31, 1606-1613 (2011).

21. Jocham, G., Klein, T. a \& Ullsperger, M. Differential Modulation of Reinforcement Learning by D2 Dopamine and NMDA Glutamate Receptor Antagonism. J. Neurosci. 34, 13151-13162 (2014).

22. Eisenegger, C. et al. Role of dopamine D2 receptors in human reinforcement learning. Neuropsychopharmacology 39, 2366-2375 (2014).

23. Swart, J. C. et al. Catecholaminergic challenge uncovers distinct Pavlovian and instrumental mechanisms of motivated (in)action. Elife 6, 1-36 (2017).

24. Frank, M. J. et al. Genetic triple dissociation reveals multiple roles for dopamine in reinforcement learning. PNAS 104, 16311-16316 (2007).

25. Jacobs, E. \& D’Esposito, M. Estrogen Shapes Dopamine-Dependent Cognitive Processes: Implications for Women's Health. J. Neurosci. 31, 5286-5293 (2011).

26. Eisenegger, C. et al. DAT1 Polymorphism Determines L-DOPA Effects on Learning about Others ' Prosociality. PLoS One 8, e67820 (2013).

27. Pessiglione, M., Seymour, B., Flandin, G., Dolan, R. J. \& Frith, C. D. Dopaminedependent prediction errors underpin reward-seeking behaviour in humans. Nature 442, 1042-1045 (2006).

28. Doll, B. B., Bath, K. G., Daw, N. D. \& Frank, M. J. Variability in dopamine genes dissociates model-based and model-free reinforcement learning. J. Neurosci. 36, 1211-1222 (2016).

29. Nemmi, F. et al. Interaction between striatal volume and DAT1 polymorphism predicts 
working memory development during adolescence. Dev. Cogn. Neurosci. 30, 191199 (2018).

30. Boksem, M. A. S., Tops, M., Kostermans, E. \& De Cremer, D. Sensitivity to punishment and reward omission: Evidence from error-related ERP components. Biol. Psychol. 79, 185-192 (2008).

31. Boksem, M. A. S., Tops, M., Wester, A. E., Meijman, T. F. \& Lorist, M. M. Errorrelated ERP components and individual differences in punishment and reward sensitivity. Brain Res. 1101, 92-101 (2006).

32. Lee, S. H., Ham, B. J., Cho, Y. H., Lee, S. M. \& Shim, S. H. Association study of dopamine receptor D2Taql A polymorphism and reward-related personality traits in healthy Korean young females. Neuropsychobiology 56, 146-151 (2008).

33. Reuter, M., Schmitz, A., Corr, P. \& Hennig, J. Molecular genetics support Gray's personality theory: The interaction of COMT and DRD2 polymorphisms predicts the behavioural approach system. Int. J. Neuropsychopharmacol. 9, 155-166 (2006).

34. Sommer, T. et al. Effects of the experimental administration of oral estrogen on prefrontal functions in healthy young women. Psychopharmacology (Berl). 235, 34653477 (2018).

35. Bayer, J., Gläscher, J., Finsterbusch, J., Schulte, L. H. \& Sommer, T. Linear and inverted U-shaped dose-response functions describe estrogen effects on hippocampal activity in young women. Nat. Commun. 9, 1-12 (2018).

36. Jakob, K., Ehrentreich, H., Holtfrerich, S. K. C., Reimers, L. \& Diekhof, E. K. DAT1genotype and menstrual cycle, but not hormonal contraception, modulate reinforcement learning: Preliminary evidence. Front. Endocrinol. (Lausanne). 9, 60 (2018).

37. Stricker, R. et al. Establishment of detailed reference values for luteinizing hormone , 
follicle stimulating hormone, estradiol, and progesterone during different phases of the menstrual cycle on the Abbott ARCHITECT $\square$ analyzer. Clin. Chem. Lab. Med. 44, 883-887 (2006).

38. Eisenegger, C., von Eckardstein, A., Fehr, E. \& von Eckardstein, S. Pharmacokinetics of testosterone and estradiol gel preparations in healthy young men. Psychoneuroendocrinology 38, 171-178 (2013).

39. Steyer, R., Schwenkmezger, P., Notz, P. \& Eid, M. Testtheoretische Analysen des Mehrdimensionalen Befindlichkeitsfragebogen. Diagnostica 40, 320-328 (1994).

40. Patton, J. H., Stanford, M. S. \& Barratt, E. S. Factor structure of the barratt impulsiveness scale. J. Clin. Psychol. 51, 768-774 (1995).

41. Carver, Charles, S. \& White, Teri, L. Behavioral Inhibition, Behavioral Activation, and Affective Responses to Impending Reward and Punishment: The BIS/BAS Scales. J. Pers. Soc. Psychol. 67, 319-333 (1994).

42. Kim, S. H., Yoon, H. S., Kim, H. \& Hamann, S. Individual differences in sensitivity to reward and punishment and neural activity during reward and avoidance learning. Soc. Cogn. Affect. Neurosci. 10, 1219-1227 (2014).

43. Sali, A. W., Anderson, B. A. \& Yantis, S. Reinforcement learning modulates the stability of cognitive control settings for object selection. Front. Integr. Neurosci. 7, 95 (2013).

44. Unger, K., Heintz, S. \& Kray, J. Punishment sensitivity modulates the processing of negative feedback but not error-induced learning. Front. Hum. Neurosci. 6, 186 (2012).

45. Lighthall, N. R., Gorlick, M. A., Schoeke, A., Frank, M. J. \& Mather, M. Stress Modulates Reinforcement Learning in Younger and Older Adults. Psychol Aging 28, 35-46 (2013). 
46. Cools, R. \& D’Esposito, M. Inverted-U-shaped dopamine actions on human working memory and cognitive control. Biol. Psychiatry 69, 113-125 (2011).

47. Heinz, A. et al. Genotype influences in vivo dopamine transporter availability in human striatum. Neuropsychopharmacology 22, 133-139 (2000).

48. Ahn, W.-Y., Haines, N. \& Zhang, L. Revealing Neurocomputational Mechanisms of Reinforcement Learning and Decision-Making With the hBayesDM Package. Comput. Psychiatry 1, 24-57 (2017).

49. Ahn, W. et al. Decision-making in stimulant and opiate addicts in protracted abstinence : evidence from computational modeling with pure users. Front. Psychol. 5, $1-15$ (2014).

50. Daw, N. D., Doherty, J. P. O., Dayan, P., Seymour, B. \& Dolan, R. J. Cortical substrates for exploratory decisions in humans. Nature 441, 876-879 (2006).

51. Smith, C. T., Sierra, Y., Oppler, S. H. \& Boettiger, C. A. Ovarian Cycle Effects on Immediate Reward Selection Bias in Humans: A Role for Estradiol. J. Neurosci. 34, 5468-5476 (2014).

52. Diekhof, E. K. Be quick about it. Endogenous estradiol level, menstrual cycle phase and trait impulsiveness predict impulsive choice in the context of reward acquisition. Horm. Behav. 74, 186-193 (2015).

53. Reimers, L., Büchel, C. \& Diekhof, E. K. How to be patient. The ability to wait for a reward depends on menstrual cycle phase and feedback-related activity. Front. Neurosci. 8, 401 (2014).

54. Chowdhury, R. et al. Dopamine restores reward prediction errors in old age. Nat. Neurosci. 16, 648-653 (2013).

55. Chowdhury, R., Guitart-Masip, M., Bunzeck, N., Dolan, R. J. \& Düzel, E. Dopamine modulates episodic memory persistence in old age. J. Neurosci. 32, 14193-14204 
(2012).

56. Männistö, P. T. \& Kaakkola, S. Catechol-O-methyltransferase (COMT): Biochemistry, molecular biology, pharmacology, and clinical efficacy of the new selective COMT inhibitors. Pharmacol. Rev. 51, 593-628 (1999).

57. Colzato, L. S. \& Hommel, B. Effects of estrogen on higher-order cognitive functions in unstressed human females may depend on individual variation in dopamine baseline levels. Front. Neurosci. 8, 65 (2014).

58. Colzato, L. S., Hertsig, G. \& Wildenberg, van den Hommel, B. Estrogen modulates inhibitory control in healthy human females: evidence from the stop-signal paradigm. Neuroscience 167, 709-715 (2010).

59. Colzato, L. S., Pratt, J. \& Hommel, B. Estrogen modulates inhibition of return in healthy human females. Neuropsychologia 50, 98-103 (2012).

60. Geniole, S. N. et al. Using a Psychopharmacogenetic Approach To Identify the Pathways Through Which—and the People for Whom-Testosterone Promotes Aggression. Psychol. Sci. 30, 481-494 (2019).

61. Losecaat Vermeer, A. B. et al. Exogenous testosterone increases status-seeking motivation in men with unstable low social status. Psychoneuroendocrinology 113, (2020).

62. Sheehan, D. V. et al. The validity of the Mini International Neuropsychiatric Interview (MINI) according to the SCID-P and its reliability. Eur. Psychiatry 12, 232-241 (1997).

63. Fishman, J., Boyar, R. M. \& Hellman, L. Influence of body weight on estradiol metabolism in young women. J. Clin. Endocrinol. Metab. 41, 989-991 (1975).

64. Schneider, J. et al. Effects of Obesity on Estradiol Metabolism: Decreased Formation of Nonuterotropic Metabolites. J. Clin. Endocrinol. Metab. 56, 973-978 (1983). 
65. Maidenbaum, S., Miller, J., Stein, J. M. \& Jacobs, J. Grid-like hexadirectional modulation of human entorhinal theta oscillations. PNAS 115, 10798-10803 (2018).

66. Eisenegger, C., Naef, M., Snozzi, R., Heinrichs, M. \& Fehr, E. Prejudice and truth about the effect of testosterone on human bargaining behaviour. Nature 463, 356-359 (2010).

67. Aeberli, I., Molinari, L. \& Zimmermann, M. B. A composite score combining waist circumference and body mass index more accurately predicts body fat percentage in 6- to 13-year-old children. Eur. J. Nutr. 52, 247-253 (2013).

68. Bolker, B. M. et al. Generalized linear mixed models: a practical guide for ecology and evolution. Trends in Ecology and Evolution vol. 24 127-135 (2009).

69. Bates, D., Mächler, M., Bolker, B. M. \& Walker, S. C. Fitting linear mixed-effects models using Ime4. J. Stat. Softw. 67, (2015).

70. Watkins, C. J. C. H. \& Dayan, P. Q-learning. Mach. Learn. 8, 279-292 (1992).

71. de Boer, L. et al. Dorsal striatal dopamine D1 receptor availability predicts an instrumental bias in action learning. PNAS 116, 261-270 (2019).

72. Rutledge, R. B. et al. Dopaminergic Drugs Modulate Learning Rates and Perseveration in Parkinson 's Patients in a Dynamic Foraging Task. J. Neurosci. 29, 15104-15114 (2009).

73. Vehtari, A., Gelman, A. \& Gabry, J. Practical Bayesian model evaluation using leaveone-out cross-validation and WAIC. Stat. Comput. 27, 1413-1432 (2017).

74. Rigoux, L., Stephan, K. E., Friston, K. J. \& Daunizeau, J. Bayesian model selection for group studies - Revisited. Neuroimage 84, 971-985 (2014).

75. Daunizeau, J., Adam, V. \& Rigoux, L. VBA: A Probabilistic Treatment of Nonlinear Models for Neurobiological and Behavioural Data. PLoS Comput. Biol. 10, (2014). 
76. Schaaf, M. E. Van Der, Fallon, S. J., Huurne, N., Buitelaar, J. \& Cools, R. Working Memory Capacity Predicts Effects of Methylphenidate on Reversal Learning. Neuropsychopharmacology 38, 2011-2018 (2013).

77. Longcope, C., Kato, T. \& Horton, R. Conversion of blood androgens to estrogens in normal adult men and women. J. Clin. Invest. 48, 2191-2201 (1969).

78. Yaffe, K. et al. Androgen receptor CAG repeat polymorphism is associated with cognitive function in older men. Biol. Psychiatry 54, 943-946 (2003).

79. Beyenburg, S. et al. Androgen receptor mRNA expression in the human hippocampus. Neurosci. Lett. 294, 25-28 (2000).

80. Kovacs, D. et al. The androgen receptor gene polyglycine repeat polymorphism is associated with memory performance in healthy Chinese individuals. Psychoneuroendocrinology 34, 947-952 (2009).

81. Gillies, G. E. \& McArthur, S. Estrogen actions in the brain and the basis for differential action in men and women: A case for sex-specific medicines. Pharmacol. Rev. 62, $155-198$ (2010).

82. Bayer, J. et al. Estrogen and the male hippocampus: Genetic variation in the aromatase gene predicting serum estrogen is associated with hippocampal gray matter volume in men. Hippocampus 23, 117-121 (2013).

83. Kravitz, H. M., Meyer, P. M., Seeman, T. E., Greendale, G. A. \& Sowers, M. F. R. Cognitive Functioning and Sex Steroid Hormone Gene Polymorphisms in Women at Midlife. Am. J. Med. 119, 94-102 (2006).

84. Almey, A., Milner, T. A. \& Brake, W. G. Estrogen receptors in the central nervous system and their implication for dopamine-dependent cognition in females. Horm. Behav. 74, 125-138 (2015).

85. Ma, S. L. et al. Polymorphisms of the estrogen receptor (ESR1) gene and the risk of 
bioRxiv preprint doi: https://doi.org/10.1101/2020 02 18 954982; this version posted May 10,2020 . The copyright holder for this preprint

(which was not certified by peer review) is the author/funder, who has granted bioRxiv a license to display the preprint in perpetuity. It is made available under aCC-BY-NC-ND 4.0 International license. 


\section{Supplementary Materials:}

\section{A causal role for estradiol in human reinforcement learning}

738 Sebastijan Veselic ${ }^{1,2,3 *}$, Gerhard Jocham ${ }^{4}$, Christian Gausterer ${ }^{5}$, Bernhard Wagner ${ }^{6}$, Miriam

739 Ernhoefer-Reßler ${ }^{6}$, Rupert Lanzenberger ${ }^{7}$, Clauss Lamm $^{1,8}$, Christoph Eisenegger $^{1}$ \& Annabel

740 Losecaat Vermeer ${ }^{1 *}$ 


\section{Subjects}

The short version of e-MINI ${ }^{62}$ was used to screen and exclude those who had a nondiagnosed, disclosed, or a diagnosed psychiatric disorder. Subjects were recruited through social media, web portals, and flyers on university premises. All subjects provided written informed consent and were financially compensated for the completion of the experiment (50€) and received an additional maximum bonus of $40 €$ (range $7 €-30 €$ ) based on their performance in the all the tasks.

\section{Measurement Instruments}

\section{Experimental Tasks}

767 For each task, we gave subjects paper instructions including control questions to check whether all subjects understood the instructions. All tasks except for the N-BACK task were monetarily incentivised.

Reinforcement Learning: In addition to the main 500 trials, subjects were trained on 10 practice trials with two initial options to learn how the task works. The initial two options were then changed (i.e. the sensory cues) before the main trials started. We did this to avoid carryover effects from practice to the main task. As shown in Fig. 1, each trial included three stages: (1) a cue onset stage $(5 \mathrm{sec})$ where subjects had to decide between the two options and press the corresponding key. If they did not respond within that time frame, they would see a warning message indicating they should respond and try to be faster next time; (2) a choice feedback stage ( $1 \mathrm{sec})$ where subjects received information about both the chosen (thick frame) and unchosen (thin frame) option (yellow - correct, red - wrong); and (3) an inter-trial interval ( $M=$ $1.5 \mathrm{sec}$, jittered between 0.9 to $2.1 \mathrm{sec})$.

Working memory capacity: As described in the manuscript, subjects were presented with a sequence of letters one-by-one. For each letter, they had to decide if the current letter was the 
same as the one presented $N$ trials ago by pressing "R", in case it was not the same they had to press "O". For example, in the 3-back condition, the letter sequence " $\boldsymbol{A} \boldsymbol{B} \boldsymbol{D} \boldsymbol{A} \boldsymbol{A}$ " would require subjects to press "R" only to the second occurrence of A, as this was the same letter as the one 3 trials ago. The last $A$ in this example sequence is defined as a lure trial, while the other letters were nontarget trials. Lure trials were present only in the 2-BACK and 3-BACK conditions as in ${ }^{25}$, and while lure trials were added to keep the task consistent with their implementation, we did not further analyse them separately as they were not relevant for our question. In total, there were four blocks per condition. Each block was announced by an instruction lasting for $2 \sec ($ Fig. 1A), a fixation cross (1 sec) and a sequence of 20 trials. Each trial was presented for $1 \mathrm{sec}$ with a $1 \mathrm{sec}$ feedback phase and a $1 \mathrm{sec}$ inter-stimulus interval. After every 20 trials, subjects had a $3 \mathrm{sec}$ resting period, before the next block was announced. A lack of response to any cue was considered a miss.

\section{Procedure}

During the first session, we assessed height, weight, abdominal, and visceral fat because these variables could impact estradiol metabolisation ${ }^{63,64}$. On the second session subjects applied a topical transparent gel on their chest and shoulders that either contained $2 \mathrm{mg}$ of estradiol (Divigel, Orion Pharma AG, Zug Switzerland) or a placebo. A male experimenter was present to ensure that the subjects applied the gel correctly.

\section{Statistical analysis}

\section{Behavioural analysis}

To quantify statistical significance for the cumulative choice difference reported in the manuscript, we employed permutation testing (2000 iterations) by shuffling the responses of each subject and thereby decoupling the label from the responses, thus building a null distribution. The null distribution shows the difference that would be expected from random allocation to the group. To determine significance, we computed z-scores as measures of standardized effect size (e.g. ${ }^{65}$ ) by subtracting from the quantity of interest the mean of the 
null distribution and dividing it by the standard deviation of the null distribution. From this, we were able to use the Fisher-z-transformation to obtain p-values.

We used two-sample proportion z-tests to determines the percentage of trials on which the number of subjects who chose option A in one group was statistically significantly different from the other group on a trial-by-trial basis.

For all general linear models reported in the main results, we regressed out several nuisance regressors known to impact estradiol metabolism or affect reinforcement learning behaviour. These included weight, $\mathrm{BMI}$, abdominal and visceral fat ${ }^{63,64}$, post-administration cortisol levels ${ }^{45}$, and beliefs related to having received the drug. This was done because of our previous work showing the impact of beliefs about a hormone on subsequent behaviour, irrespective of whether subjects underwent treatment or received placebo ${ }^{66}$.

All linear models were compared with BIC and AIC. Unless stated otherwise in the main text, for all reported results the winning model regressed out cortisol levels following administration, beliefs about having received the drug, the certainty of that belief and whether they had observed any changes in themselves, a composite score of weight and BMI that were summed together ${ }^{67}$ because of their high intrinsic correlation $(r=0.89)$, visceral, and abdominal fat. For general linear models involving accuracy, we also regressed out reaction times to control for accuracy-speed trade-offs. All nuisance regressors were z-scored.

We compared both treatment groups for age and other bodily characteristics (i.e. BMI, height, weight, visceral, and abdominal fat) and potential differences in self-reported mood (MDBF), impulsiveness (BIS-11) and reward responsiveness (BIS/BAS) (see Questionnaires, Table S4 and S5). We used two-tailed independent samples Welch t-tests, or Wilcoxon signed-rank test if assumptions of normality were not met, to test whether the groups matched on all variables. To test for mood differences after administration between the treatment groups, we performed an ANCOVA for each of the three subscales of the MDBF questionnaire where we controlled for baseline mood scores. Two-way ANOVAs were further performed on 
the individual subscales of the BIS-11 questionnaire to investigate whether there was an interaction between the group (estradiol, placebo) and session (pre, post) on impulsiveness. target accuracy, reaction times, and d-prime. We analyzed this with an ANOVA containing the together with an interaction term for group and condition. the Ime4 package in $\mathrm{R}^{68,69}$ to investigate whether the interaction between drug, DAT1 or

842 COMT and trial would be predictive of the subjects' chosen option.

\section{Computational modelling}

844 To test whether estradiol would increase reward sensitivity and thereby learning, we

845 formalized behaviour within a reinforcement learning framework and fitted several Q-learning models ${ }^{70}$ with softmax choice rules:

Q-learning model (equation 1):

$$
Q_{t+1}^{A}=Q_{t}^{A}+\alpha\left(R_{t}^{A}-Q_{t}^{A}\right)
$$

Softmax choice rule (equation 2):

$$
p=\frac{1}{1+e^{\tau\left(Q_{t}^{A}-Q_{t}^{B}\right)}}
$$

Where, $t$ is time, $A$ is option A, $Q$ is subjective value, $\alpha$ is the learning rate, $R$ is the obtained reward, and $\tau$ is the temperature parameter. Equations 1 and 2 represent our first model (model 1). In Q-learning, the basic idea is that agents learn subjective values of actions they perform in their environment. Subjective values are learned and updated through a value function (Equation 1) following feedback after each action. A teaching signal known as the learning rate-weighted prediction error dictates how strongly the subjective value will be updated on each action. The prediction error corresponds to the difference between the obtained and expected reward (i.e. the subjective value prior to making the new choice). Within 
this process, the learning rate dictates how heavily new information will be weighted in proportion to previous information about the option, and therefore how strongly the subjective value will change from its current estimate. The softmax equation then yields the probability of selecting an action given the learning rate and the temperature parameter, which reflects stochasticity of choice behaviour.

By employing computational modelling of this sort, we were able to obtain parameter estimates that quantify the difference in subjects' behaviour, captured by a difference in learning rates. To obtain a more precise account of the effect of estradiol on reward processing, we extended the basic Q-learning model in several ways, as described below.

The first extension (equation $3 a$ and $3 b$ ) allowed for separate learning rates for $\alpha_{\text {Pos }}$ and $\alpha_{\text {Neg }}$ that would differentiate between learning from positive and negative prediction errors.

$$
\begin{aligned}
& Q_{t+1}^{A}=Q_{t}^{A}+\alpha_{\text {Pos }}\left(R_{t}^{A}-Q_{t}^{A}\right) \\
& Q_{t+1}^{A}=Q_{t}^{A}+\alpha_{N e g}\left(R_{t}^{A}-Q_{t}^{A}\right)
\end{aligned}
$$

The updating with a positive learning rate occurs when the prediction error term $\left(R_{t}^{A}-Q_{t}^{A}\right)$ evaluates to positive while updating with the negative learning rate occurs when the prediction error term evaluates to negative. Furthermore, due to reward stochasticity of our task reward probability distribution (obtained by a Gaussian random walk - Fig. 1B), we added an additional parameter $\xi$, representing a lapse or irreducible noise parameter ${ }^{71}$ in our choice rule (equation 4):

$$
p=\frac{1}{1+e^{\tau\left(Q_{t}^{A}-Q_{t}^{B}\right)}}(1-\xi)+\frac{\xi}{2}
$$

Finally, we added a perseverance parameter $\lambda^{72}$ (equation 5): 


$$
p=\frac{1}{1+e^{\tau\left(Q_{t}^{A}-Q_{t}^{B}+\lambda C\right)}}(1-\xi)+\frac{\xi}{2}
$$

Where $C=1$, if the same option was chosen on trial $n$ and trial $n+1$, and $C=-1$ if the opposite was true. In summary, our full model had separate learning rates for positive and negative prediction errors, a choice stochasticity, irreducible noise, and perseverance parameter. All other models were reduced cases of this model and all possible combinations of the described free parameters therefore yielded eight models in total for which we estimated parameters:

Table S0. Models and parameters

\begin{tabular}{ll}
\hline Model 1 & $\begin{array}{l}\text { Learning rate, temperature } \\
\text { Model } 2\end{array}$ \\
Model 3 & Learning rate, temperature, lapse \\
Model 4 & Learning rate, temperature, lapse, perseverence \\
Model 5 & Positive and negative learning rate, temperature \\
Model 6 & Positive and negative learning rate, temperature, lapse \\
Model 7 & Positive and negative learning rate, temperature, perseverence \\
Model 8 & Positive and negative learning rate, temperature, lapse, perseverence
\end{tabular}

891

The model fitting was performed using JAGS and the rjags ( $v 4.9$ ) package in $R(v$ 3.6.0). Each model was run with 5000 samples each with 1000 burn-in samples on three chains. Priors over parameters and hyperparameters were set to default as described in ${ }^{48}$. We computed the leave one out information criterion using the loo package ${ }^{73}$ and used this metric to compare the models. Furthermore, we performed Bayesian model comparison by computing the (protected) exceedance probability ${ }^{74}$ using the VBA toolbox ${ }^{75}$ to determine the best model and compare its congruency with the LOOIC measure. Finally, we extracted the posterior predictive density for each subject as a measure of predictive power of the best model. This was then compared to the actual behaviour as a measure of static (accuracy 
901

902

903

904

905

906

907

908

909

910

911

912

913

914

915

916

917

918

919

920

921

922

923

924

925

collapsed across time) and dynamic (accuracy at each trial across subjects) predictive accuracy. From the obtained maximum a-posteriori estimates we then generated synthetic datasets for each subject and refit the model using synthetic data to assess parameter recovery. We did this by correlating our original and recovered parameters to determine whether the learning rate parameters of the winning model were correlated.

\section{Genotyping}

\section{DNA extraction and quantification}

Buccal swabs were collected using sterile cotton swabs (Sarstedt AG, Germany). DNA was extracted from swabs using the QIAamp DNA Mini kit (Qiagen, Hilden, Germany) and eluted in a final volume of $50 \mu \mathrm{L}$ of QIAamp buffer AE (Qiagen). Human nuclear DNA was quantified using the Applied Biosystems (AB) 7500 real-time PCR instrument (Thermo Fisher Scientific, Waltham, MA) and the Quantifiler Human Plus quantification Kit (AB) following manufacturer's recommendations.

\section{Typing of repeat length polymorphisms}

Genomic DNA fragments that contain polymorphic repeat sequences were amplified in two separate reactions: i.e. a multiplex PCR (simultaneously targeting $A R(C A G) n, D A T 1$ VNTR, Era(TA)n and $\operatorname{Er}(C A) n$ ) and a singleplex PCR (targeting solely $A R(G G N) n)$, respectively.

The multiplex PCR was performed using $5 \mathrm{ng}$ template DNA in a reaction mix (total volume of $25 \mu \mathrm{L}$ ) consisting of $1 \times$ GeneAmp PCR buffer $(\mathrm{AB}), 0.25 \mathrm{mM}$ each dNTP, 2.5 units AmpliTaq Gold polymerase (AB) and target specific primers (AR(CAG), DAT1, ERa and Er $\beta$; including 5'-fluorescent-dye-labeled forward primers; details provided in Table 1). The following protocol was applied using the Veriti 96 -well thermal cycler $(A B): 35$ cycles at $95^{\circ} \mathrm{C}$ for 30 seconds, $55^{\circ} \mathrm{C}$ for 1 minute, and $72{ }^{\circ} \mathrm{C}$ for 1 minute. Before the first cycle, an initial denaturation ( $95^{\circ} \mathrm{C}$ for 5 minutes) was included, and the last cycle was followed by a final extension step at $72^{\circ} \mathrm{C}$ for 45 minutes. 
bioRxiv preprint doi: https://doi org/10.1101/2020.02 18.954982 this version posted May 10.2020 . The copyright holder for this preprin (which was not certified by peer review) is the author/funder, who has granted bioRxiv a license to display the preprint in perpetuity. It is made available under aCC-BY-NC-ND 4.0 International license.

The singleplex PCR was conducted using $5 \mathrm{ng}$ template DNA in a reaction mix (total volume of $20 \mu \mathrm{L})$ containing target specific primers (AR(GGN)n, details provided in Table S1)), $0.5 \mu \mathrm{L}$ Phire Hot Start II DNA polymerase (Thermo Fisher) in $1 \times$ Phire reaction buffer (Thermo Fisher). Amplification was carried out on the Veriti thermal cycler (AB) and included an initial denaturation step at $98^{\circ} \mathrm{C}$ for 30 seconds, followed by 33 cycles of 10 seconds at $98^{\circ} \mathrm{C}, 30$ seconds at $60{ }^{\circ} \mathrm{C}$ and 30 seconds at $72{ }^{\circ} \mathrm{C}$. The last cycle was followed by a final extension at $72{ }^{\circ} \mathrm{C}$ for 10 minutes.

Aliquots of PCR products were diluted with Hi-Di formamide $(A B)$, mixed with internal lane standard LIZ 600 v.2 (AB) and separated on the ABI 3500 Genetic Analyzer applying standard conditions. The number of repeats predicted by the GeneMapper ID-X software (AB) was in full agreement to the actual repeats determined by direct sequencing of PCR products using the BigDye Terminator Sequencing Kit v3.1 (AB) in selected DNA samples.

Table SI. Panel of loci and primer sets used for the typing of repeat length polymorphisms

\begin{tabular}{|c|c|c|c|c|c|}
\hline Marker & Location ${ }^{b}$ & Primer sequence $5^{\prime}-3^{\prime}$ c & Dye & Orientation & $\begin{array}{l}\text { Conc. } \\
\text { (nM) }\end{array}$ \\
\hline \multirow[t]{2}{*}{$A R(G G N) n^{a}$} & $\begin{array}{l}\text { chrX:67546447- } \\
67546603(+)\end{array}$ & CCTGGCACACTCTCTTCACA & VIC & forward & 625 \\
\hline & & GTTTCTGGCCGAGTGTAGCCGTAG & & reverse & \\
\hline \multirow[t]{2}{*}{$\mathrm{AR}(\mathrm{CAG}) \mathrm{n}$} & $\begin{array}{l}\text { chrX:67545237- } \\
67545434(+)\end{array}$ & CGCGAAGTGATCCAGAACC & 6-FAM & forward & 200 \\
\hline & & GTTTCTAGAACCATCCTCACССТGCT & & reverse & \\
\hline \multirow[t]{2}{*}{$\begin{array}{l}\text { DAT1 } \\
\text { VNTR }\end{array}$} & $\begin{array}{l}\text { chr5:1393559- } \\
1394008(-)\end{array}$ & TGTGGTGTAGGGAACGGCCTGAGA & 6-FAM & forward & 400 \\
\hline & & TGTTGGTCTGCAGGCTGCCTGCAT & & reverse & \\
\hline \multirow[t]{2}{*}{$\mathrm{ERa}(\mathrm{TA}) \mathrm{n}$} & $\begin{array}{l}\text { chr6:151806472- } \\
151806594(+)\end{array}$ & AACTATCCAAGATTATAGACGCATGA & NED & forward & 600 \\
\hline & & GTTTCTAACATGCACACGCACATACA & & reverse & \\
\hline $\operatorname{Er} \beta(C A) n$ & $\begin{array}{l}\text { chr14:64253529- } \\
64253650(-)\end{array}$ & GTGCTGCGAGCAGAGATA & PET & forward & 800 \\
\hline
\end{tabular}




\section{Typing of the COMT Val158Met polymorphism}

950 SNaPshot minisequencing was applied for the typing of Val158Met variants in the COMT

951 gene. Therefore, a $177 \mathrm{bp}$ fragment of genomic DNA harbouring the causative single 952 nucleotide polymorphism (SNP rs4680) in its centre was amplified by PCR. The reaction mix 953 comprised $5 \mathrm{ng}$ template DNA, $1 \times$ GeneAmp PCR buffer $(\mathrm{AB}), 0.25 \mathrm{mM}$ each dNTP, 2.5 units

954 AmpliTaq Gold polymerase (AB) and target specific primers (details provided in Table S2) in 955 a total reaction volume of $25 \mu \mathrm{L}$. Thermal cycling was performed applying the Veriti cycler (AB) 956 and conditions as follows: $95^{\circ} \mathrm{C}$ for $5 \mathrm{~min}$; 35 cycles of $95^{\circ} \mathrm{C}$ for 15 seconds, $59{ }^{\circ} \mathrm{C}$ for 30 seconds and $72^{\circ} \mathrm{C}$ for 1 minute; final extension at $72{ }^{\circ} \mathrm{C}$ for 5 minutes.

Table S2. Primer set used for PCR of the COMT fragment

\begin{tabular}{lllll}
\hline Marker & Location $^{\mathbf{a}}$ & Primer sequence 5'-3' $\mathbf{b}$ & Orientation & $\begin{array}{l}\text { Conc. } \\
(\mathbf{n M})^{\mathbf{c}}\end{array}$ \\
\hline COMT & $\begin{array}{l}\text { chr22:19963623- } \\
19963799(+)\end{array}$ & GGGCCTACTGTGGCTACTCA & forward & 400 \\
& & & &
\end{tabular}

\section{GCCCTTTTTCCAGGTCTGA reverse}

${ }^{a}$ Chromosome number and genomic location of targeted sequence (orientation provided in brackets) according to UCSC version hg38 (http://genome.ucsc.edu/).

961

PCR products were purified from excess primers and dNTPs by ExoSAP-IT (Thermo Fisher) treatment following manufacturer's recommendations. Minisequencing was conducted on a

Veriti thermal cycler $(\mathrm{AB})$ in a total volume of $10 \mu \mathrm{L}$ containing $3 \mu \mathrm{L}$ of purified PCR product,

Table S3. Minisequencing primer information

\begin{tabular}{|c|c|c|c|}
\hline $\begin{array}{l}\text { SNP } \\
\text { sequence } \\
\text { variation }\end{array}$ & Location ${ }^{\mathrm{a}}$ & Primer sequence $5^{\prime}-3^{\prime}$ b & Orientation \\
\hline$G>A$ & $\begin{array}{l}\text { chr22:19963728- } \\
19963747\end{array}$ & 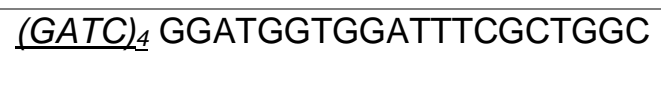 & forward \\
\hline
\end{tabular}

970 a Chromosome number and genomic location of primer binding site (orientation provided in brackets) according to 971 UCSC version hg38 (http://genome.ucsc.edu/). The targeted SNP rs4680 is located at position chr22:19963748. 
b The non-specific primer tail is underlined in Italics

ExoSAP-IT treatment was again applied for the clean-up of the minisequencing reaction. $5 \mu \mathrm{l}$ of purified minisequencing reaction product was then mixed with $9.3 \mu \mathrm{L} \mathrm{Hi-Di}$ formamide $(\mathrm{AB})$ and $0.2 \mu \mathrm{L}$ of GeneScan-LIZ 120 internal size standard (AB). After a denaturing step for 5 min at $98^{\circ} \mathrm{C}$ followed by cooling to $4{ }^{\circ} \mathrm{C}$ the fragments were separated on an ABI PRISM 310 Genetic Analyzer (AB) with POP4 polymer and analysed with GeneMapper v3.2 software. Calling of SNP variants based on minisequencing was in full agreement to results from direct sequencing of PCR products in selected DNA samples.

\section{Hormone concentrations}

Quantification of estrone and estradiol in saliva samples was performed with derivatization using pentafluorobenzoyl chloride (PFBCI) and the addition of the isotopically labeled internal standards estrone- $\mathrm{d}_{4}$ and estradiol- $\mathrm{d}_{5}$. Organic saliva was reacted with $1.0 \mathrm{~mL} 1 \% \mathrm{PFBCl}$ and $0.1 \mathrm{~mL}$ pyridine at $60^{\circ} \mathrm{C}$ for $30 \mathrm{~min}$. The derivatization agents were evaporated, the sample was reconstituted with $0.5 \mathrm{~mL} \mathrm{NaHCO} 3$ and extracted with $1 \mathrm{~mL} \mathrm{n}$-hexane. The organic phase was substituted with $0.2 \mathrm{~mL}$ dodecane and subjected to optimized GC-MS/MS analysis using an Agilent $7890 \mathrm{GC}$ with Agilent DB-17ht $15 \mathrm{~m} \times 0.25 \mathrm{~mm} \times 0.15 \mu \mathrm{m}$ capillary column connected to an Agilent 7010 tandem mass spectrometer operated in MRM mode using negative chemical ionization at $150^{\circ} \mathrm{C}$ with methane as a reaction gas $(40 \%, 2 \mathrm{~mL} / \mathrm{min})$. Method validation was performed using ion transition $\mathrm{m} / \mathrm{z} 464$-> 400 as a quantifier for estrone and m/z 660 -> 596 for estradiol, whereas a LLOQ of $1.92 \mathrm{fg}$ o.c. and $1.94 \mathrm{fg}$ was obtained, respectively.

Quantification of hydrocortisone and testosterone in saliva samples was performed using liquid chromatography tandem mass spectrometry (LCMS/MS), with an Agilent 6460 with electrospray ionization in positive mode coupled to a 1290 UHPLC system. Collision energy was optimized for specific MRM transitions of Hydrocortisone (363.2/121.1 m/z; 363.2/91.1 m/z), Testosterone (289.2/109.1; 289.2/97.1 m/z), 2,3,4-13C3-Hydrocortisone (366.2/124 m/z) and 2,3,4-13C3-Testosterone (292.2/100 m/z). Agilent Poroshell 120 EC-C18 
1000

1001

1002

1003

1004

1005

1006

1007

1008

1009

1010

1011

1012

1013

1014

1015

1016

1017

1018

1019

1020

1021

1022

1023

1024

1025

was used for chromatographic separation under reversed phase conditions. The internal standard preparation and internal standard mixture was prepared containing 2,3,4-13C3Hydrocortisone; 2,3,4-13C3-Testosterone, 2,4,16,16,17-d5-17b-Estradiol and concentration of $5 \mathrm{ng} / \mathrm{mL}$ each. Samples were prepared by adding $100 \mu$ internal standards $(5 \mathrm{ng} / \mathrm{mL})$ to $500 \mu \mathrm{l}$ plasma or saliva and the steroids were extracted using $4 \mathrm{~mL}$ MTBE. After $10 \mathrm{~min}$. overhead shacking, the samples were centrifuged for $5 \mathrm{~min}$. at $3000 \mathrm{rpm}$ and the top MTBE layer was transferred to a test tube. MTBE was evaporated using a centrivap concentrator at $40^{\circ} \mathrm{C}$ (Labconco). The residual sample was then re-dissolved in methanol and analyzed by LC-MS/MS.

\section{Questionnaires}

Mood: To control for a potential confound of mood, tiredness, or alertness from the treatment affecting subjects' performance ${ }^{22}$, we assessed subjects' self-reported mood before and after administration of the treatment, using the German Multidimensial Mood State Questionnaire (“Der Mehrdimensionale Befindlichskeitfragebogen - MDBF) ${ }^{39}$ Both versions of this questionnaire (A and B) contain 12 items with a 5-level Likert scale and three subscales that test for different continuums of mood (Good-Bad [ $\left.\alpha_{\text {pre }}=.81, \alpha_{p o s t}=.77\right]$, Awake-Tired [ $\alpha_{\text {pre }}=$ $\left..84, \alpha_{\text {post }}=.87\right]$, Calm-Nervous [ $\left.\alpha_{\text {pre }}=.73, \alpha_{\text {post }}=.75\right]$ ).

Impulsiveness: We used the Barratt Impulsiveness Scale (BIS-11; 40 to measure subjects' impulsiveness as ${ }^{52}$ observed that variations in estradiol levels differentially affected women with low trait as opposed to high trait impulsiveness. BIS-11 is a widely used measure for impulsiveness with 30 items describing common behaviour and preferences related to (non)impulsiveness which individuals have to rate on a 4-point scale (1 - rarely/never, almost always/always -4$)$. The General Impulsiveness $\left(\alpha_{\text {pre }}=.71, \alpha_{\text {post }}=.75\right)$ factor together with its three second-order factors (Motor Impulsiveness ( $\alpha_{\text {pre }}=.47, \alpha_{\text {post }}=.54$ ) Nonplanning Impulsiveness $\left(\alpha_{\text {pre }}=.6, \alpha_{\text {post }}=.63\right)$, Attentional Impulsiveness $\left(\alpha_{\text {pre }}=.49, \alpha_{\text {post }}=.52\right)$ are reported. 
1026 Behavioural inhibition and activation: we measured the trait behavioural activation and 1027 inhibition with the Behavioural inhibiton/Behavioural Activation Scales (BIS/BAS; ${ }^{41}$. The BAS 1028 scale is a 24-item questionnaire answered on a four-level scale (1-very true for me, 4 - very false for me). It is subdivided into Drive $(\alpha=.74)$, Fun Seeking $(\alpha=.67)$, and Reward Responsiveness $(\alpha=.6)$ while the BIS scale $(\alpha=.77)$ is unidimensional. Drive is thought to measure the persistent pursuit of goals (e.g. "I go out of my way to get the things I want"), Fun Seeking: the desire for new rewards and willingness to approach events that would be potentially rewarding (e.g. "I crave excitement and new sensations"), while Reward Responsiveness focuses on positive responses that would occur if a reward is anticipated (e.g. "When I am doing well at something I love to keep doing it"). Finally, the BIS scale measures sensitivity to negative events (e.g. "Criticism or scolding hurts me quite a bit").

\section{Belief probes}

In addition, we probed subjects' beliefs and confidence about receiving estradiol (e.g. whether they believed they received estradiol or a placebo, how certain they were of this answer, and whether they noticed any changes). This was done to later regress out the potential contribution of beliefs arising, for example, from subjects researching potential side effects of the hormone prior the experiment. Namely, subjects' beliefs about having received a hormone and beliefs about the effects of a hormone on their performance have previously shown to modulate behaviour independent of whether subjects had actually received $\mathrm{it}^{66}$. 


\section{Results}

1054

1055

1056

1057

1058

1059

1060

1061

1062

1063

1064

1065

1066

1067

1068

1069

1070

1071

1072

1073

1074

1075

1076

1077

1078

1079

\section{Matching of both groups}

In the first part of the supplementary results, Table S4 and S5 show that our random assignment was successful as the groups did not differ in any of the measured parameters before (Table S4) administration and as a function of administration (Table S5). However, we did observe the expected change in estradiol metabolite concentrations in the estradiol group, outlined below.

\section{Hormone concentrations}

We observed a statistically significant post-administration difference between both groups in log-transformed estradiol concentrations $(W=1545,95 \% \mathrm{Cl}[0.03,1.87], p<.05)$ with the estradiol group having higher estradiol metabolite concentration following administration (estradiol: $M d n=41.77 \pm 531.54$ ), placebo: $M d n=5.55 \pm 230.23$ ) but not before (estradiol: $M d n$ $=3.38 \pm 230.97)$, placebo: $M d n=1.89 \pm 21.92)$ compared to the placebo group $(W=1498$, $95 \% \mathrm{Cl}[-0.05,1.03], p=.09)$. We report the median for the values above because even after log-transforming the metabolite concentrations, they were not distributed normally. Importantly, because we have observed high interindividual variance in estradiol concentrations prior to administration, we have reason to believe the obtained metabolite concentrations were contaminated during the handling of the samples following our data collection. Namely, in previous work such baseline variation was not observed despite an identical procedure and dosage with the main difference being that serum levels of estradiol were measured there ${ }^{38}$. Log-transformed estrone and cortisol concentrations after administration were also examined showing no differences between both groups. Estrone: (experimental: $M d n=8.79 \pm 4226.69)$, control: $M d n=5.80 \pm 161.99)(W=1427,95 \% \mathrm{Cl}[-0.17$, 1.05], $p=.16)$, cortisol: (experimental: $M d n=0.77 \pm 0.94)$, control: $M d n=0.73 \pm 1.15)(W=$ $1207,95 \% \mathrm{Cl}[-0.31,0.27], p=.90)$. 


\section{Bodily measures and behavioural characteristics}

1081 As outlined in Table S4, both the estradiol and placebo group were also matched for their 1082 weight, height, BMI, visceral, abdominal fat, and individual sub scales of the BIS/BAS

1083

1084

1085

1086

1087

1088

1089

1090

1091

1092

1093

1094

1095

1096

1097

1098

questionnaire (Drive, Reward, Fun-Seeking, Behavioural Inhibition). Similarly, separate oneway ANOVAs revealed no interaction for the four subscales of BIS-11 (Table S5) (General: $F_{(1,195)}=0.01, p=0.91$, Attentional: $F_{(1,195)}=0.04, p=.85$, Motor: $F_{(1,195)}=0.59, p=.45$, nonplanning: $\left.F_{(1,195)}=0.08, p=.78\right)$.

Furthermore, we checked whether both the estradiol and placebo group did not differ in pre-existing differences in working memory (Figure S2A, S2B, S2C) in addition to testing whether administration influenced mood (Figure S2D). By doing so we were able to exclude differences in working memory and mood leading to the observed results ${ }^{26,76}$. Separate ANCOVAs for the three subscales (Alertness, Mood, Calmness) of the MDBF revealed no differences in post-administration (Post) scores between the estradiol and placebo group when controlling for baseline scores (Pre) as a covariate (Mood: $F_{(1,96)}=0.30, p=0.58$; Alertness: $F_{(1,96)}=1.35, p=.25$; Calmness: $\left.F_{(1,96)}=1.34, p=.25\right)$. Similarly, we observed no interaction between group membership and post-administration score (Mood: $F_{(1,96)}=0.06, p$ $=.81$; Alertness: $F_{(1,96)}=1.88, p=.17$; Calmness: $\left.F_{(1,96)}=1.55, p=.22\right)$.

Table S4: Descriptive statistics by treatment (Estradiol, Placebo).

\begin{tabular}{lccccc}
\hline \multicolumn{1}{c}{ Group } & & & \\
& $\begin{array}{c}\text { Estradiol } \\
(\mathbf{n = 5 0})\end{array}$ & $\begin{array}{c}\text { Placebo } \\
(\mathbf{n = 5 0})\end{array}$ & $\boldsymbol{n}$ & statistic [95\% Cl] & $\boldsymbol{p}$ \\
\hline Age (years) & $25.12(3.63)$ & $24.6(3.44)$ & 100 & $1381[-0.99,1.49]^{1}$ & 0.99 \\
BMI & $24.54(2.65)$ & $24.35(3.08)$ & 99 & $1286[-0.99,1.99]^{1}$ & 0.99 \\
Height (cm) & $181.90(6.88)$ & $180.40(5.95)$ & 99 & $1.16[-1.07,4.07]$ & 0.94 \\
Weight (kg) & $81.09(9.66)$ & $79.48(11.44)$ & 99 & $0.76[-2.61,5.83]$ & 0.99 \\
Visc. Fat (\%) & $6.20(2.48)$ & $6.06(2.90)$ & 98 & $1248[-0.99,1.00]^{1}$ & 0.99 \\
Abd. Fat (\%) & $20.66(5.97)$ & $19.74(6.32)$ & 98 & $0.74[-1.55,3.38]$ & 0.99 \\
\hline
\end{tabular}


bioRxiv preprint doi: https://doi.org/10.1101/2020.02 18.954982; this version posted May 10, 2020. The copyright holder for this preprint (which was not certified by peer review) is the author/funder, who has granted bioRxiv a license to display the preprint in perpetuity. It is made available under aCC-BY-NC-ND 4.0 International license.

\section{BIS/BAS}

\begin{tabular}{lccccc}
\hline BIS & $17.54(2.80)$ & $18.40(3.68)$ & 100 & $-1.32[-1.86,-0.25]$ & 0.88 \\
Drive & $11.72(1.77)$ & $12.12(2.32)$ & 100 & 1074.5 & 0.91 \\
Reward & $11.62(1.99)$ & $12.46(2.04)$ & 100 & $921^{1}$ & 0.18 \\
Fun Seeking & $15.88(2.00)$ & $16.08(2.17)$ & 100 & $-0.48[-1.03,0.63]$ & 0.99
\end{tabular}

1099 Note: Values in cells denote M, parentheses denote SD. The superscript 1 denotes the Mann-Whitney-Wilcoxon $1100 \quad W$ value. For the remaining group comparisons, two-tailed independent samples Welch $t$-tests were employed. In 1101 cases where $n$ is not equal to $N=100$, data was not recorded for that particular variable. $p$-values are Bonferroni 1102 corrected. 

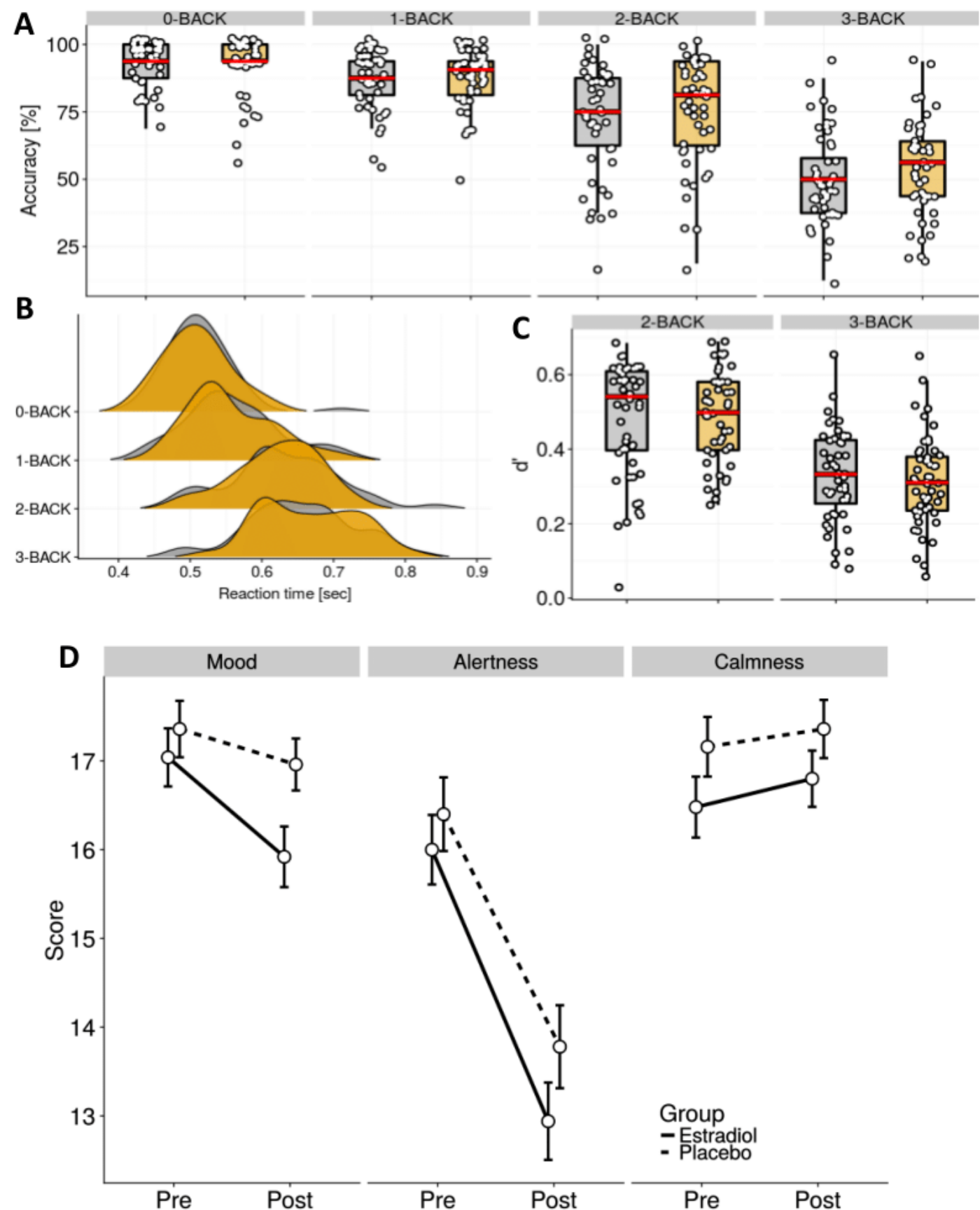

Figure S1. A) Accuracy for individual conditions. The red bar represents the median, the box plot represents the $75 \%$ middle most data points, with the whiskers representing $1.95^{\star} I Q R$. Orange depicts the estradiol and gray the placebo group. That is, they represent the division of subjects according to whether they would subsequently be allocated to the estradiol or placebo group. This color convention is used throughout all figures. B) shows density plots for reaction time data for individual conditions. C) shows d' in the most difficult two conditions (2-BACK, 3$\mathrm{BACK})$ as there were no false alarms in the 0-BACK and 1-BACK, thus accuracy is reduced to d'. D) Average scores prior and post administration for the three subscales of the MDBF. 
1115 Furthermore, our working memory (N-BACK) task revealed a comparable picture for accuracy

1116 (Figure S2A), reaction times (Figure S2B), and d-prime (Figure S2C). That is, there was no

1117 statistically significant difference between the estradiol and placebo group in accuracy,

1118 average reaction times, and d-prime. We did observe an expected drop in performance in

1119 terms of decreased accuracy (0-BACK: $92.94 \pm 9.34,1-B A C K: 88.06 \pm 10.78,2-B A C K: 74.25$

$1120 \pm 19.38,3$-BACK: $51.56 \pm 17.37$ ), and d-prime (2-BACK: $0.48 \pm 0.14,3$-BACK: $0.32 \pm 0.12$ ),

1121 and increased reaction times (0-BACK: $0.51 \pm 0.05,1$-BACK: $0.56 \pm 0.06,2-B A C K: 0.63 \pm$

$11220.07,3$-BACK: $0.66 \pm 0.07$ ) as the condition became more difficult (i.e. went from 0-BACK to

1123 3-BACK). Separate linear models were used to compute to check for main effects of drug $\left(F_{(1,}\right.$

1124 196) $=2.01, p=.16)$ and an interactive effect of drug and condition on d-prime $\left(F_{(1,196)}=0.82\right.$,

$1125 p=.37$ ). As mentioned above, we also did this for accuracy (main effect of drug: $F_{(1,392)}=1.07$,

$1126 p=.30$; drug ${ }^{*}$ condition interaction: $F_{(3,392)}=2.30, p=.08$ ), and reaction times (main effect:

$1127 \quad F_{(1,347)}=1.31, p=.25 ;$ drug $^{*}$ condition interaction: $\left.F_{(1,347)}=0.99, p=.39\right)$.

Table S5. Descriptive statistics of MDBF and BIS-11 subscales.

\begin{tabular}{lccccc}
\hline & \multicolumn{2}{c}{ Pre-administration } & \multicolumn{2}{c}{ Post-administration } \\
MDBF & $\boldsymbol{N}$ & Estradiol & Placebo & Estradiol & Placebo \\
\cline { 2 - 6 } & & & & \\
\hline Mood & 100 & $17.04(2.31)$ & $17.36(2.25)$ & $15.92(2.41)$ & $16.96(2.07)$ \\
Alertness & 100 & $16.00(2.77)$ & $16.40(2.93)$ & $12.94(3.08)$ & $13.78(3.30)$ \\
Calmness & 100 & $16.48(2.43)$ & $17.16(2.38)$ & $16.80(2.24)$ & $17.36(2.32)$ \\
\hline
\end{tabular}

BIS-11

\begin{tabular}{llllll}
\hline General & 100 & $57.86(7.68)$ & $59.28(7.45)$ & $59.96(8.51)$ & $60.46(7.86)$ \\
Motor & 100 & $20.76(3.00)$ & $21.78(3.42)$ & $22.02(3.42)$ & $22.42(3.65)$ \\
Attention & 100 & $14.34(3.37)$ & $14.14(2.17)$ & $15.10(3.30)$ & $14.64(2.68)$ \\
Nonplanning & 100 & $22.76(3.61)$ & $23.36(4.29)$ & $22.84(4.05)$ & $23.40(4.21)$ \\
\hline
\end{tabular}

We observed no correlation between the certainty in the subjects' belief as to whether they had received the drug or placebo $(r=0.02, p=.82)$, or between the reported observed changes and actually receiving estradiol $(r=-0.08, p=.42)$. This shows that our double-blind procedure was successful, and that our placebo gel preparation was indistinguishable from the actual drug. 
Table S6. Frequencies of individual polymorphisms of DAT and COMT genes.

\begin{tabular}{lll}
\hline Polymorphism & Group & N \\
\hline $9 / 10$ & Estradiol & 18 \\
$9 / 10$ & Placebo & 16 \\
$10 / 10$ & Estradiol & 21 \\
$10 / 10$ & Placebo & 26 \\
Val/Val & Estradiol & 11 \\
Val/Val & Placebo & 9 \\
Met/Val & Estradiol & 23 \\
Met/Val & Placebo & 26 \\
Met/Met & Estradiol & 12 \\
Met/Met & Placebo & 15
\end{tabular}

Note: the split according to both COMT and DAT does not sum to 100 because for a few subjects it was not possible to determine their polymorphism.

\section{Reinforcement learning task}

The role of CYP 19A1, ERa, ER $\beta, C A G$, and GGN

1144 Because the results for accuracy, staying, and switching behaviour that we report in the manuscript could also be moderated through other candidate mechanisms, we further analysed these candidate mechanisms together by providing theoretical motivation for the analyses. Here, we first briefly outline their importance and then summarize the observed results.

It is known that androgens are converted to estrogen ${ }^{77}$. This means that the increase in estrogen levels arises from this conversion process and the administration more directly. Furthermore, variation in the length of two functional polymorphisms (CAG - polyglutamine, and GGN - polyglycine) are known to modulate the functioning of the androgen receptor gene 78. This is important for two reasons. First, our procedure has previously shown to increase circulating testosterone levels which could have raised estradiol levels whilst being moderated by subjects' androgen receptor characteristics ${ }^{38}$. Following from this, previous work has shown that brain regions important for memory and learning contain androgen receptors ${ }^{79}$. 
Therefore, it could be possible that interindividual differences in both functional polymorphisms could have moderated our observed results due to interindividual variability. For example, greater CAG repeat length has previously been associated with lower scores in different cognitive tests in older men ${ }^{78}$, and GGN repeat length has been associated with immediate and delayed logical memory recall in women ${ }^{80}$. The described results show a correlation between individual variability in androgen receptor functioning and cognitive performance, giving rise to the possibility that CAG and GGN polymorphisms being potential candidate mechanisms moderating the observed effect of estradiol.

Throughout the conversion process from androgens to estrogens, the CYP19A1 gene encodes instructions for aromatase - the enzyme converting androgens to estrogens ${ }^{81}$. The single nucleotide polymorphisms (SNPs) associated with the CYP19A1 gene regulate the metabolism of androgens and mediate brain estrogen activity. Two specific SNPs (rS700518, rs936306) have been previously shown to have a role in cognitive functioning in humans. For example, men with the homozygous AA allele have higher estradiol serum levels and greater bilateral posterior hippocampal gray matter volume compared to men with the homozygous GG allele ${ }^{82}$. While other work has shown a differential impact of homozygous CC alleles versus homozygous TT alleles on episodic memory recall in women ${ }^{83}$. Given that our procedure has previously shown to increase circulating testosterone levels and that polymorphisms of the CYP19A1 gene are known to have a role in cognitive functioning, we aimed to exclude the possibility that this may have driven our observed effects, and analysed both single nucleotide polymorphisms of the CYP19A1 gene.

Once androgens are converted to estrogens, estrogen action is mediated through the estrogen receptors (ERa, ER $\beta)$. Both receptors are widely distributed throughout the brain, including regions of importance for cognitive functioning and reward processing ${ }^{84}$. So far, it has been shown that ERa is responsible for most of estrogen-related activation. For example, it has been shown that SNPs of ERa are related to Alzheimer's disease and are associated with the likelihood of developing cognitive impairment ${ }^{85}$. We have, therefore, focussed on two particular SNPs of ERa: rs9340799, rs2234693. In contrast, little is known of a potential impact 
of ER $\beta$. As an exploratory measure, we have included repeats of this receptor in our analysis as well.

Of the described candidates (CAG, GGN, CYP 19A1, ER $\alpha, E R \beta)$, no test revealed any effect of interest. There was no interaction between drug group (i.e. estradiol or placebo) and either the SNPs of ERa: $r s 9340799\left(F_{(2,84)}=0.66, p=.52\right)$, rs2234693 $\left(F_{(2,84)}=0.63, p=.53\right)$ in relation to accuracy. Furthermore, the same was true for the interaction between CAG repeats and drug group $\left(F_{(1,87)}=0.45, p=.51\right)$, GGN repeats and drug group $\left(F_{(1,87)}=1.31, p\right.$ $=.26)$, and SNPs of the CYP19A1 gene and drug group ( $r$ 700518 $F_{(2,84)}=1.84, p=.15$, rs936306 $\left.F_{(2,84)}=0.34, p=.72\right)$. In a final examination, we also looked at the repeats of ER $\beta$ to determine whether this could have driven any of the observed effects. However, this was not the case for either recorded variant of $\operatorname{ER} \beta\left(\operatorname{ER} \beta 1: F_{(1,87)}=0.02, p=.89\right.$, ERß2: $F_{(1,87)}=$ $0.00, p=.96)$.

Identical results were obtained for switching behaviour. While we observed a statistically significant interaction between estradiol administration and the COMT polymorphism (see next section), this was not true for any of the other mechanistic explanations. That is, no model showed an interaction between drug group and either of the SNPs of ERa: rs9340799 $\left(F_{(2,84)}=2.90, p=.06\right)$, rs2234693 $\left(F_{(2,84)}=2.88, p=.06\right)$, CAG repeats $\left(F_{(1,87)}=0.10, p=.76\right)$, GGN repeats $\left.F_{(1,87)}=1.32, p=.25\right)$, and SNPs of the CYP19A1 gene (rs700518 $F_{(2,84)}=1.81, p=.17$, rs936306 $\left.F_{(2,84)}=1.08, p=.35\right)$ in relation to switching behaviour. As in the case of accuracy, we also looked at the repeats of ER $\beta$. Again, there was no statistically significant contribution to switching behaviour from this predictor for either recorded variant of $\operatorname{ER} \beta\left(\operatorname{ER} \beta 1: F_{(1,87)}=3.05, p=.08 ; \operatorname{ER} \beta 2: F_{(1,87)}=0.96, p=.33\right)$.

We finally repeated the set of analyses for staying behaviour with no effects found of any of these variables: SNPs of ERa: rs9340799 $\left(F_{(2,84)}=1.69, p=.19\right)$, rs2234693 $\left(F_{(2,84)}=\right.$ $1.79, p=.17)$, CAG repeats $\left(F_{(1,87)}=0.38, p=.54\right)$, GGN repeats $\left.F_{(1,87)}=0.30, p=.59\right)$, SNPs of the CYP19A1 gene (rs700518 $F_{(2,84)}=1.27, p=.29$, rs936306 $\left.F_{(2,84)}=0.59, p=.55\right)$, and variant of $\operatorname{ER} \beta\left(\operatorname{ER} \beta 1: F_{(1,87)}=1.35, p=.25 ; \operatorname{ER} \beta 2: F_{(1,87)}=0.86, p=.36\right)$. 
In brief, we have shown that the effects related to accuracy, switching, and staying

1213

1214

1215

1216

1217

1218

1219

1220

1221

1222

1223

1224

1225

1226

1227

1228

1229

1230

1231

1232

1233

1234

1235

1236

1237

reported in the manuscript did not depend on (1) the overall androgen receptor functioning assessed by CAG and GGN repeat polymorphisms, (2) interindividual variability in the androgen-to-estrogen conversion process via the CYP19A1 gene polymorphism, and (3) estrogen receptor polymorphisms (ER $\alpha)$ or repeats $(E R \beta)$.

\section{The effect of estradiol administration on choice behaviour is moderated by polymorphisms of both COMT and DAT1}

We directly tested whether the effect of estradiol on choice behaviour is moderated by polymorphisms of dopamine-related genes (e.g. COMT, DAT1) by using generalized linear mixed models. We specifically tested whether the interaction between drug, polymorphism (COMT or DAT1), and trial are a significant predictor of the options they would choose.

Based on the inverted U-shape dopamine hypothesis ${ }^{46}$, we predicted that estradiol administration would upregulate reward sensitivity in subjects with low prefrontal dopaminergic activity (i.e. Val/Val) but would not, or would even impair it, in those with high prefrontal dopaminergic activity (i.e. Met/Met). The model predictions support this hypothesis as it predicted that subjects with a Met/Val $(\beta=0.20 \pm 0.04,95 \% C l[0.11,0.28], z=4.56, p<.001)$ and $\mathrm{Val} / \mathrm{Val}$ genotype $(\beta=0.37 \pm 0.06,95 \% \mathrm{Cl}[0.26,0.48], z=6.99, p<.001)$ were more likely to select option A as trials progressed when they received estradiol (Fig. S2, S4). Option A was the more rewarding option throughout the task (percent trials rewarded: $M_{\text {optionA }}=$ $\left.53.70 \%, M_{\text {optionB }}=42.91 \%\right)$.

Based on the prediction that estradiol indirectly increases striatal dopamine levels, leading to higher reward prediction errors, we expected that subjects with the 9/10 genotype (i.e. high striatal dopamine) would select the higher value option more often, while this would be less often true for subjects with the 10/10 genotype (i.e. low striatal dopamine). This prediction was supported by the model showing that subjects with the $10 / 10$ genotype with 
bioRxiv preprint doi: https://doi org/10.1101/2020.02 18.954982; this version posted May $10,2020$. The copyright holder for this preprint (which was not certified by peer review) is the author/funder, who has granted bioRxiv a license to display the preprint in perpetuity. It is made available under aCC-BY-NC-ND 4.0 International license.

placebo $(\beta=-0.12 \pm 0.04,95 \% \mathrm{Cl}[-0.04,-0.20], z=-3.03, p<.01)$ were the most likely to select the lower valued option A throughout task progression, while estradiol administration dampened this slope in subjects with the same 10/10 genotype (Fig. S4). Results from both generalized linear mixed effects models showed that once individual variation was considered, the effect of estradiol administration on choice behaviour across trials was moderated by striatal (DAT1) and prefrontal (COMT) polymorphisms.
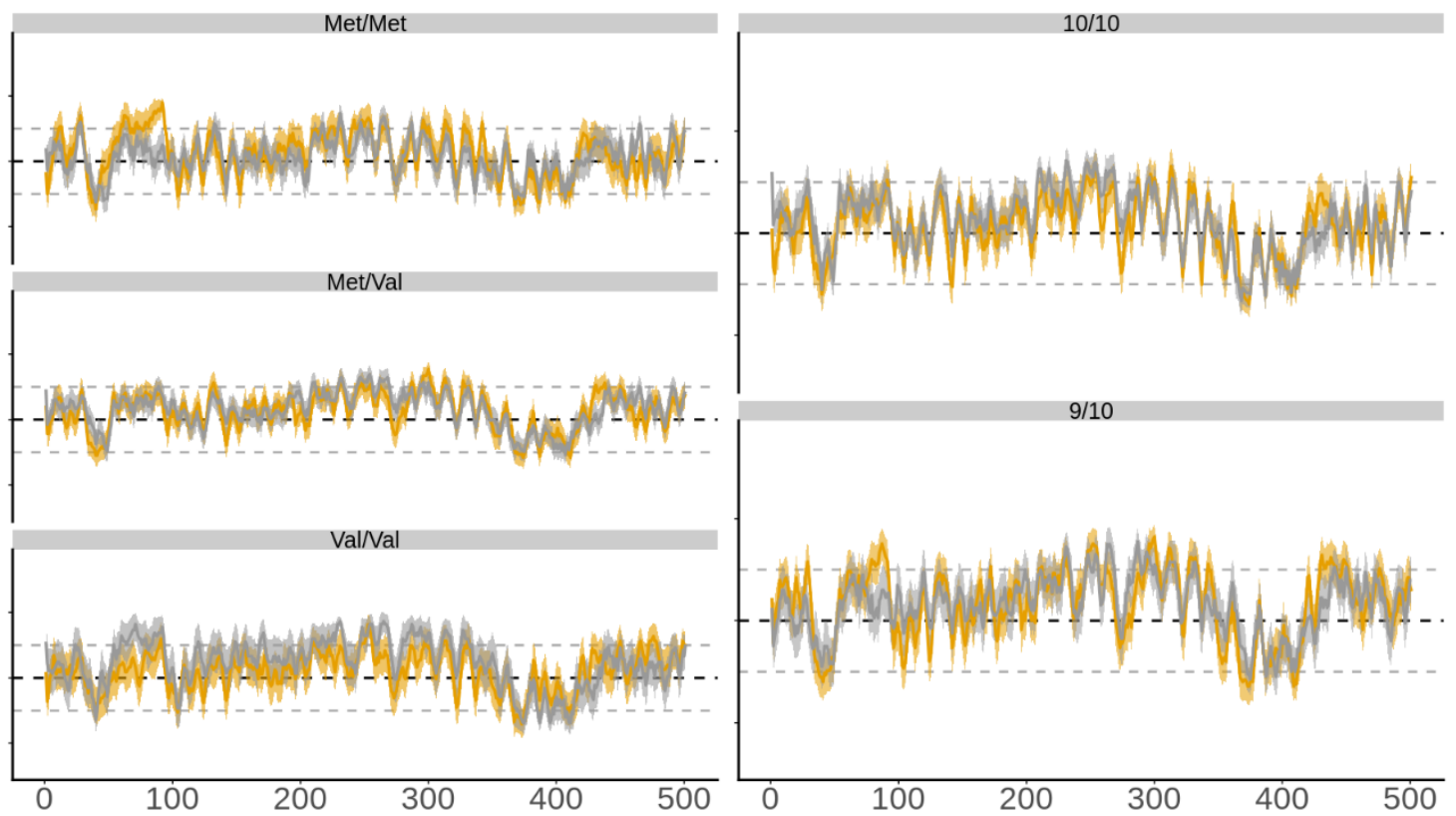

Figure S2. Relative choice probability for choosing option A (top of y-axis) vs. choosing stimulus 1 (bottom of yaxis) for the placebo (gray) and estradiol (orange) group split according to both polymorphisms assessed in the main text: COMT (left panel), DAT (right panel) across trials (1-500). Thick lines represent trial means, shaded areas denote standard error of the means. The blue line in the background denotes the empirical relative reward probability which was computed from the probability of stimulus two being rewarding (top of y-axis) - stimulus one being rewarding (bottom of y-axis). Gray dotted lines represent where subjects were on average $25 \%$ more likely to select option A (upper line) or stimulus 1 (lower line). All time-series traces are smoothed with a 5-trial moving average for visual purposes.

Figure S2 reveals a differential effect of estradiol administration on choice behaviour that depends on polymorphisms of both COMT and DAT. In the case of the COMT polymorphism this is most clearly visible in the lower left panel. The panel shows that placebo Val/Val subjects exhibited a clear tendency towards option A until trial $\sim 370$. After this, they did not reverse back towards choosing it more often despite option A being more rewarding from trial $\sim 420$ onwards. This is in contrast with results for subjects with other polymorphisms of COMT and results when subjects were split according to the DAT1 polymorphism. Estradiol 
bioRxiv preprint doi: https://doi org/10.1101/2020.02 18.954982; this version posted May 10, 2020. The copyright holder for this preprint (which was not certified by peer review) is the author/funder, who has granted bioRxiv a license to display the preprint in perpetuity. It is made available under aCC-BY-NC-ND 4.0 International license.

1261

1262

1263

1264

1265

1266

1267

1268

1269

1270

1271

1272

1273

1274

1275

1276

1277

1278

1279

1280

1281

1282

1283

1284

Met/Met subjects exhibited choice behaviour more aligned with the reward probability distribution in the beginning at trial $~ 80$ compared to subjects from the placebo group with the same polymorphism. When we then split subjects according DAT1 polymorphism, the estradiol $9 / 10$ subjects can similarly be seen following the reward probability distribution more closely compared to the placebo $9 / 10$.

Because of the results above and because estradiol administration likely results in increased prefrontal dopamine levels through downregulating COMT enzyme activity ${ }^{56}$ we predicted that the interaction between estradiol administration and COMT polymorphism would be predictive of switching behaviour ${ }^{24}$. As a measure of switching, we assessed the number of times the option chosen on trial $t$ was different from the one chosen at trial $n+1$ (i.e. a switch), irrespective of the choice outcome on trial $t$. Estradiol administration did not significantly influence switch decisions $(M=162.12 \pm 56.31)$ compared to placebo $(M=168.82$ $\pm 68.13)$. However, we observed a significant interaction of estradiol administration by COMT genotype $\left(F_{(2,80)}=3.22, p=.05, \Omega^{2}=0.04\right.$, Fig. S3). The interaction showed that subjects with placebo and a Val/Val genotype (i.e. low prefrontal dopamine availability) switched less often $(\beta=-84.07 \pm 33.69, p=.02)$ compared to all other groups. As predicted by the inverted Ushaped relationship between prefrontal dopamine levels and behaviour, Val/Val placebo subjects (Val/Val: $M=132.33 \pm 61.40)$ switched less compared to Met/Met placebo subjects (i.e. associated with high prefrontal dopamine availability; Met/Met: $M=204.27 \pm 53.52, t_{(15.10)}$ $=2.91,95 \% \mathrm{Cl}[19.25,124.54], p=.01, d=1.46)$. For the estradiol group, this difference was not present $\left(\mathrm{Val} / \mathrm{Val}: M=151.09 \pm 70.85\right.$; $\mathrm{Met} /$ Met: $M=178.5 \pm 55.34 ; t_{(18.96)}=1.03,95 \% \mathrm{Cl}[-$ 28.28, 83.10], $p=.32, d=0.44)$. In other words, estradiol administration attenuated naturally occurring differences in switching behaviour found in subjects with the Met/Met and Val/Val genotypes that are associated with high and low prefrontal dopamine levels, respectively. 
bioRxiv preprint doi: https://doi.org/10.1101/2020.02.18.954982; this version posted May 10, 2020. The copyright holder for this preprint (which was not certified by peer review) is the author/funder, who has granted bioRxiv a license to display the preprint in perpetuity. It is made available under aCC-BY-NC-ND 4.0 International license.

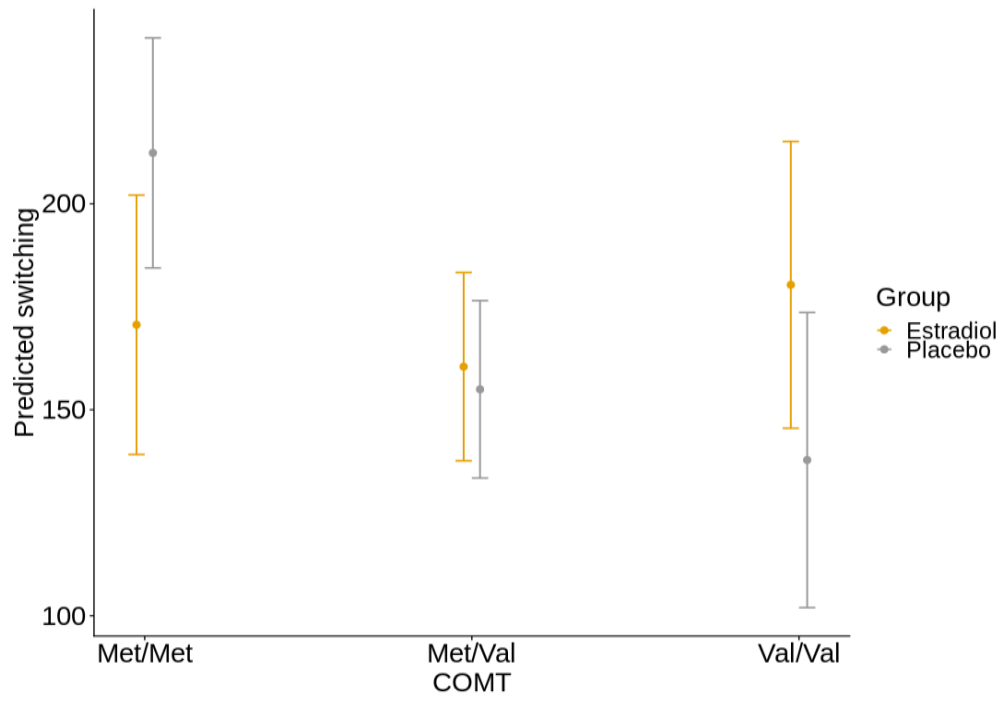

Figure S3. General linear model prediction for switching behaviour (i.e. a change in chosen stimulus on trial $t+1$ from trial $t$, independent of choice outcome on trial $t$ ). Estradiol administration dampened naturally occurring differences in switching behaviour when subjects were split according to the COMT polymorphism, i.e. whether subjects would switch the stimulus they chose on trial t compared to trial $t+1$ irrespective of choice outcome on trial $t$. Error bars represent SEM. 
bioRxiv preprint doi: https://doi org/10.1101/2020.02 18.954982; this version posted May 10,2020 . The copyright holder for this preprint (which was not certified by peer review) is the author/funder, who has granted bioRxiv a license to display the preprint in perpetuity. It is made available under aCC-BY-NC-ND 4.0 International license.

A

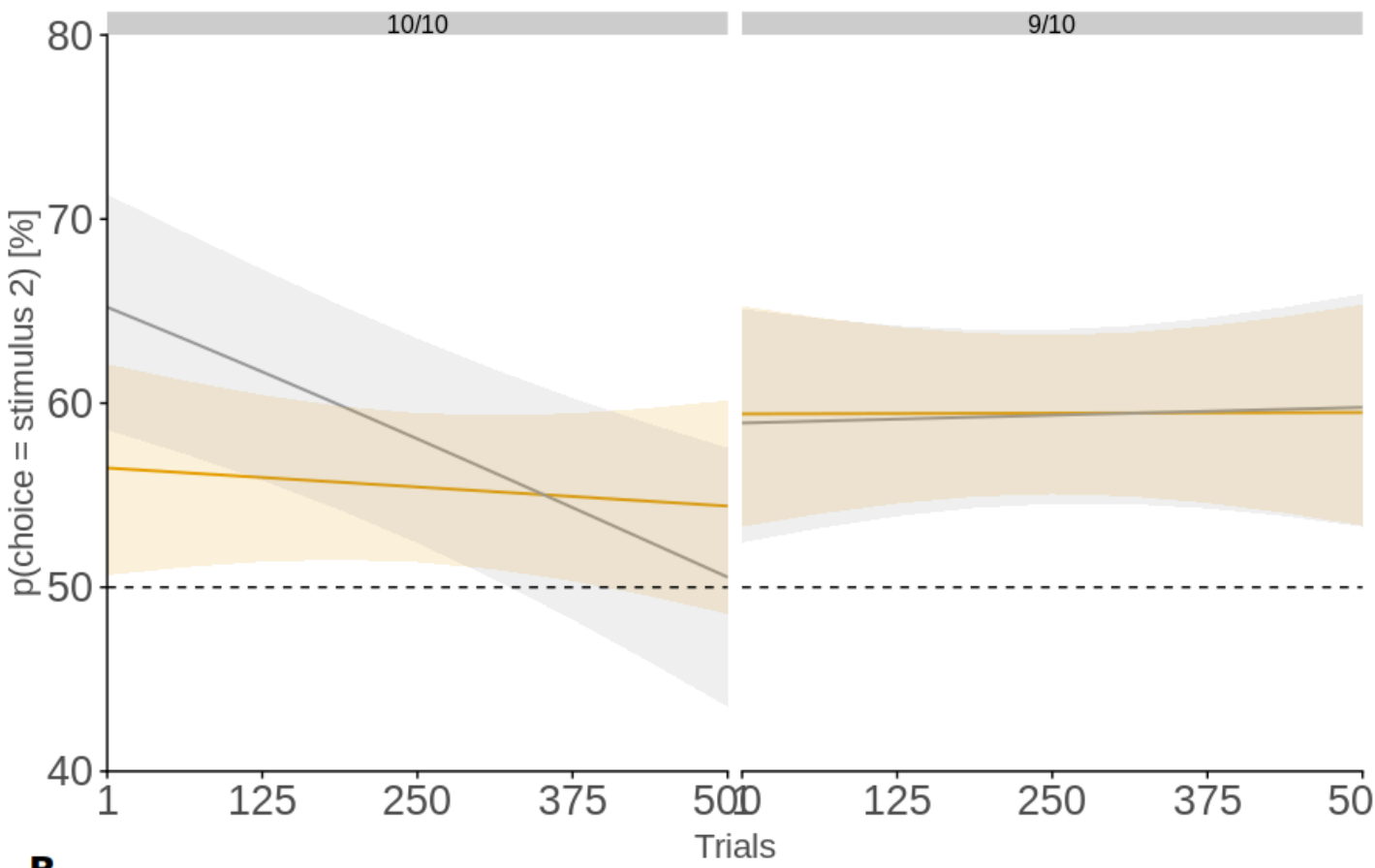

B

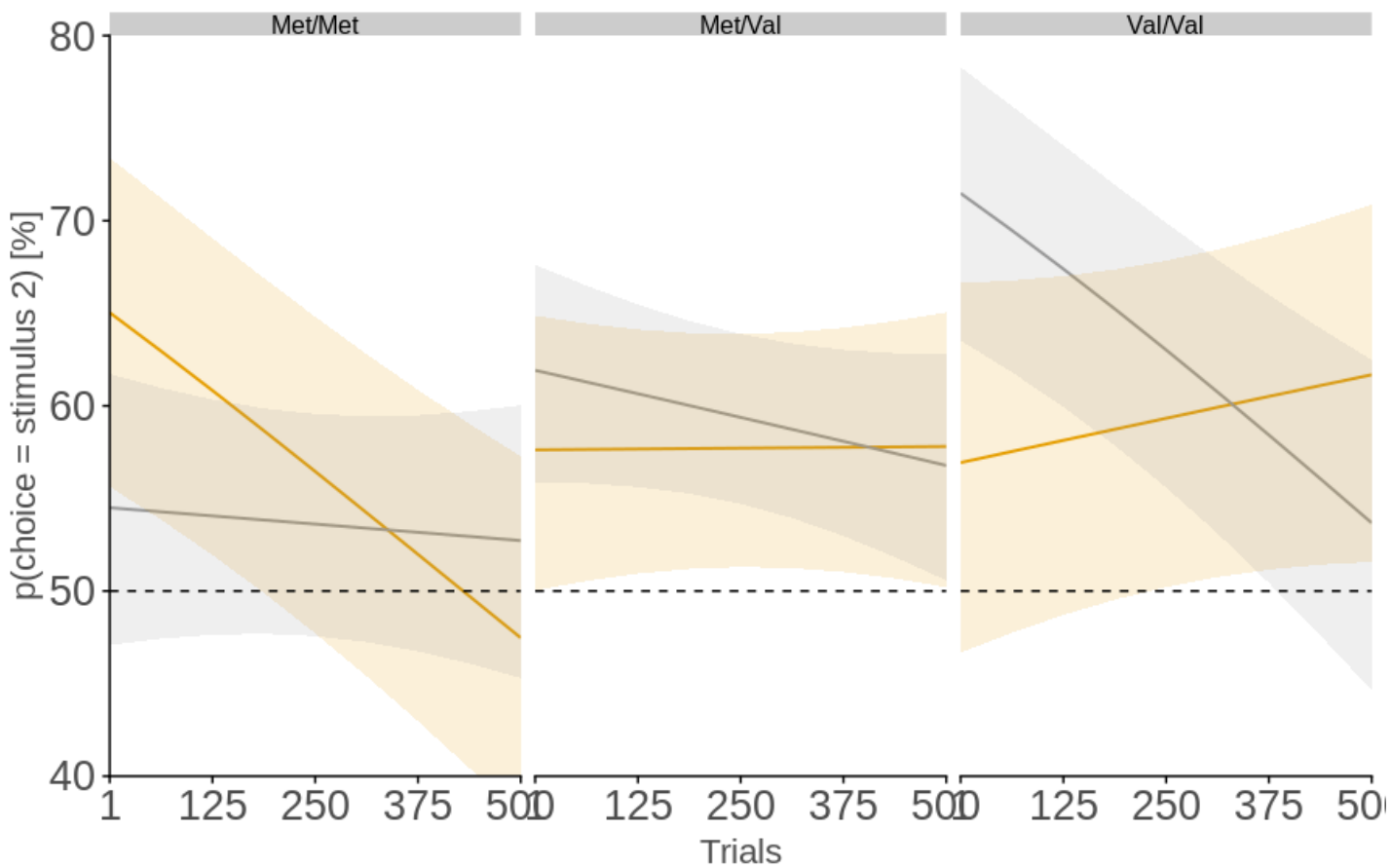

Figure S4. Predictions from winning models of the generalized linear mixed effects models for $\mathbf{A}$ ) the interaction between drug, DAT, and trial on choice, and B) the interaction between drug, COMT, and trial on choice. 
bioRxiv preprint doi: https://doi org/10.1101/2020.02 18.954982; this version posted May 10, 2020. The copyright holder for this preprint (which was not certified by peer review) is the author/funder, who has granted bioRxiv a license to display the preprint in perpetuity. It is made available under aCC-BY-NC-ND 4.0 International license.

1300

1301

1302

1303

1304

1305

1306

1307

1308

1309

1310

1311

1312

1313

1314

1315

1316

1317

\section{Formal model comparison}

We estimated parameters by fitting several Q-learning models. The best model (model 6, leave one out information criterion $(\mathrm{LOOIC})=58888$, Fig. S5A) included separate learning rates for positive and negative prediction errors, a temperature parameter, and an irreducible noise parameter. The model predicted choice behaviour above chance $\left(t_{(99)}=13.95,95 \% \mathrm{Cl}\right.$ $[0.64,0.68], p<.001$, Fig. S5B) and performed equally well for both groups $\left(M_{\text {Estradiol }}=66.26\right.$ $\left.\% \pm 10.77, M_{\text {Placebo }}=64.90 \% \pm 11.85 ; t_{(97.115)}=0.76,95 \% C l[-0.03,0.06], p=.45\right)$.

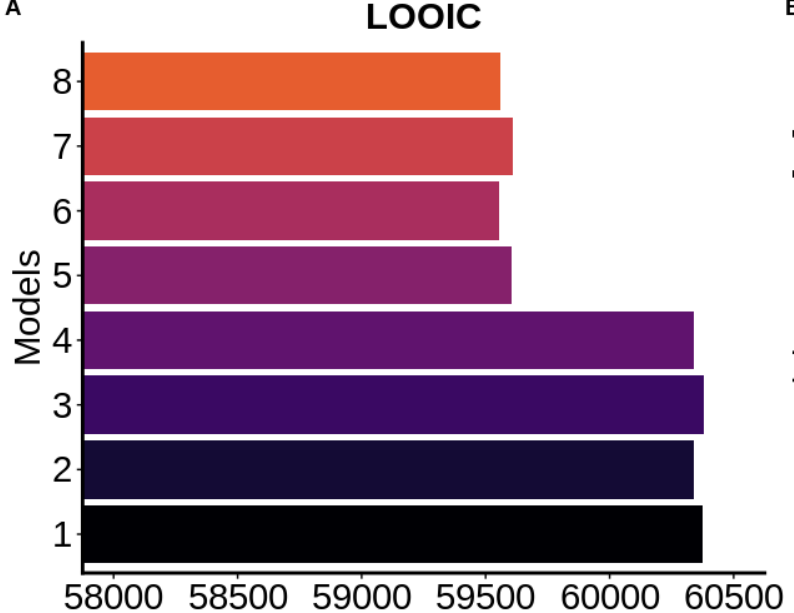

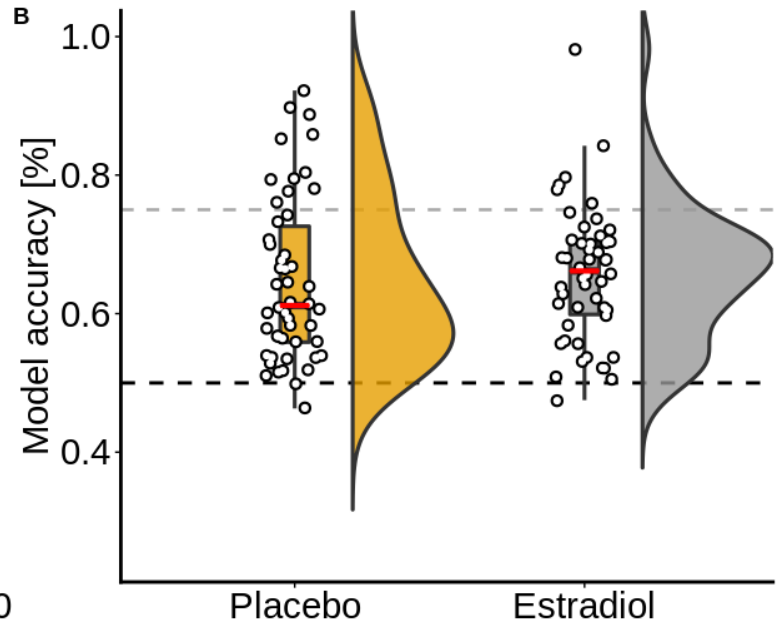

Figure S5. A) Leave one out information criterion (LOOIC) value for all employed models. Lower LOOIC indicates better model fit - model two was selected as the best model. B) The overall model accuracy collapsed over time obtained from the posterior predictive density shown for both drug groups separately.

In addition to computing the leave-one-out information criterion to perform model comparison ${ }^{73}$ we similarly computed the exceedance probability of the winning model using the VBA toolbox ${ }^{75}$. This value showed a strong preference for the winning model $P$ (model two $=98 \%$. Furthermore, we computed protected exceedance probability ${ }^{74}$ as an extension which, while yielding an expected decrease in the winning model probability, still favoured model two over other competing models $(P($ model two $)=12.5 \%)$. The likely decrease was due to the reinforcement learning task not being optimized to detect behavioural differences between the models tested. However, in all reported models, the latent variable of interest, i.e. the learning rate, remained unaltered. We would therefore expect the increase in learning 
rates to be present if we were to select the learning rates from models that best fit individual

1323 subjects.

\section{Validating model}

We further tested the model validity and predictions by computing posterior predictive densities, i.e. what predictions does the model make on a trial-by-trial basis for subjects with the parameters such as those that were extracted from our subjects. Posterior predictive densities showed no difference in a fit between both the estradiol and placebo group and approximated the empirical reward probability distribution (Fig. S6A). To quantify this, we then compared model predictions from posterior predictive densities with actual subject behaviour to assess model accuracy collapsed across time (Fig. S6B) showing it performed above chance and equally well for both groups. We further compared accuracy on each trial across subjects to ensure that there were no unexpected drops in accuracy. This did not happen as the model (Fig. S6B) had no discernible drops in performance. We also performed parameter recovery on the winning model where we used the maximum a posteriori estimates reported in the manuscript, generated data for synthetic subjects, performed parameter estimation on

1337 the synthetic data, and correlated the newly obtained synthetic parameters with original parameters for each subject. This procedure showed that both original and recovered learning rates correlated with one another (negative: $r=0.33, p<.001$, positive: $r=-0.34, p<.001$ ). 
bioRxiv preprint doi: https $/ /$ doi org/10.1101/2020.02 18 954982; this version posted May 10,2020 . The copyright holder for this preprint (which was not certified by peer review) is the author/funder, who has granted bioRxiv a license to display the preprint in perpetuity. It is made available under aCC-BY-NC-ND 4.0 International license.

1341

1342

1343

1344

1345

1346

1347

1348

1349

1350

1351

1352

1353

1354

1355

1356

1357

1358

1359

1360

1361

1362

1363

1364

1365
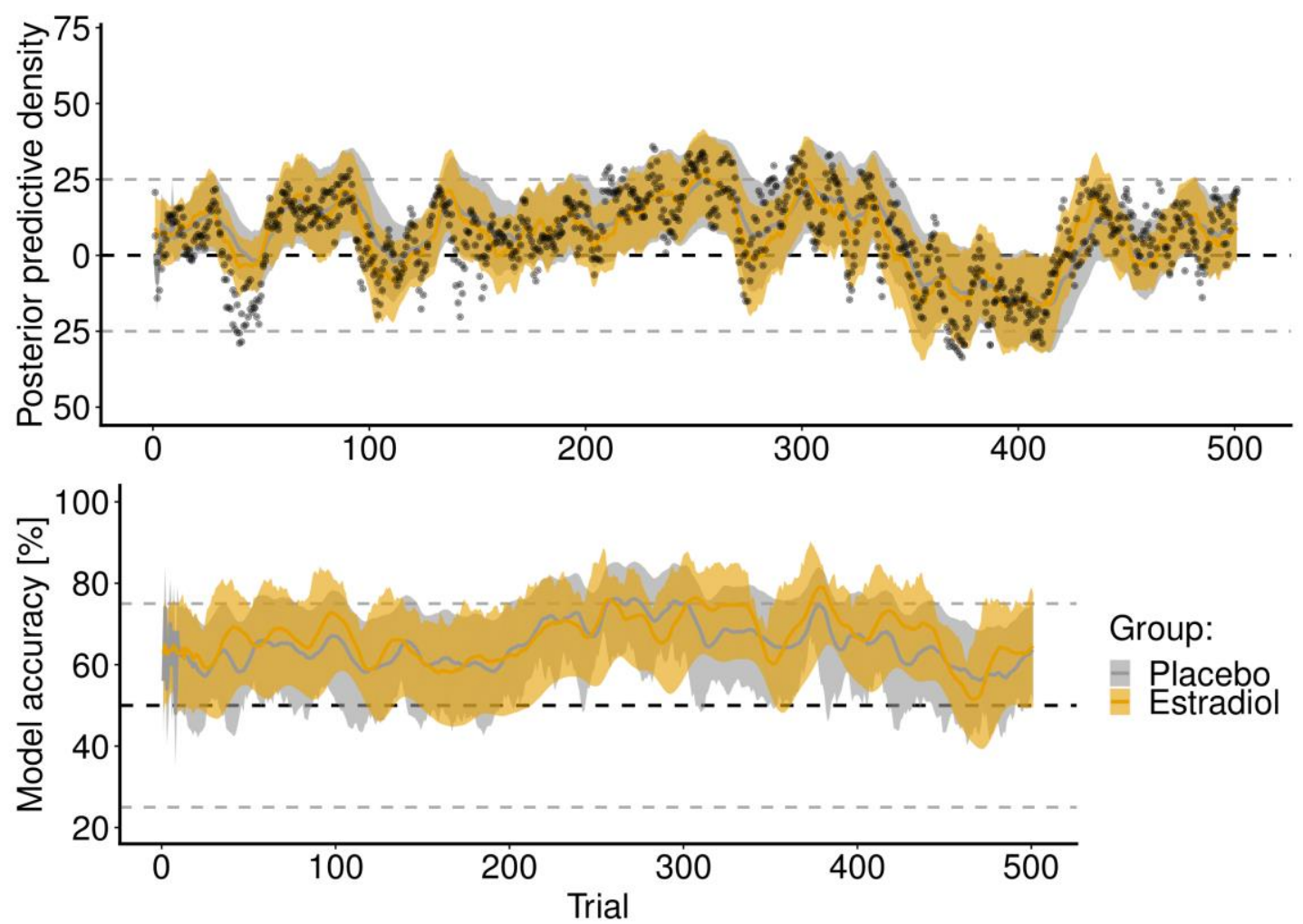

Figure S6. A) Posterior predictive density computed for both drug groups with overlaid average responses for both drug groups across trials B) Accuracy for both drug groups obtained from the posterior predictive density for both drug groups separately. 